\title{
Remarks on the Nonlocal Dirichlet Problem
}

\section{Tomasz Grzywny ${ }^{1}$ • Moritz Kassmann² $•$ Łukasz Leżaj $^{1}$}

Received: 8 August 2018 / Revised: 9 December 2019 / Accepted: 22 December 2019 /

Published online: 6 February 2020

(C) The Author(s) 2020

\begin{abstract}
We study translation-invariant integrodifferential operators that generate Lévy processes. First, we investigate different notions of what a solution to a nonlocal Dirichlet problem is and we provide the classical representation formula for distributional solutions. Second, we study the question under which assumptions distributional solutions are twice differentiable in the classical sense. Sufficient conditions and counterexamples are provided.
\end{abstract}

Keywords Dirichlet problem · Nonlocal operator · Lévy process · Interior $C^{2}$ regularity

Mathematics Subject Classification (2010) 35B65 · 35C15 · 47G20 · 60J45

\section{Introduction}

The aim of this article is to provide two results on translation-invariant integrodifferential operators, which are not surprising but have not been systematically covered in the literature. Let us briefly explain these results in case of the classical Laplace operator.

The classical result of Weyl says the following. Assume $D \subset \mathbb{R}^{d}$ is an open set, $f \in$ $C^{\infty}(D)$, and $u \in \mathcal{D}^{\prime}(D)$ is a Schwartz distribution satisfying $\Delta u=f$ in the distributional sense, i.e. $\langle u, \Delta \psi\rangle=\langle\psi, f\rangle$ for every $\psi \in C_{c}^{\infty}(D)$. Then $u \in C^{\infty}(D)$ and $\Delta u=f$ in $D$.

Research supported in part by National Science Centre (Poland) grant 2014/14/M/ST1/00600 and by the DFG through the CRCs 701 and 1283

Łukasz Leżaj

lukasz.lezaj@pwr.edu.pl

Tomasz Grzywny

tomasz.grzywny@pwr.edu.pl

Moritz Kassmann

moritz.kassmann@uni-bielefeld.de

1 Faculty of Pure and Applied Mathematics, Wrocław University of Science and Technology, 27 Wybrzeże Wyspiańskiego, Wrocław, 50-370, Poland

2 Fakultät für Mathematik, Universität Bielefeld, Postfach 100131, Bielefeld, 33501, Germany 
This is the starting point for our analysis. The first aim is to study distributional solutions to nonlocal boundary value problems of the form

$$
\begin{aligned}
\mathscr{L} u=f & \text { in } D, \\
u=g & \text { in } D^{c},
\end{aligned}
$$

where $\mathscr{L}$ is an integrodifferential operator generating a unimodal Lévy process. Our second aim is to provide sufficient conditions such that distributional solutions $u$ to the nonlocal Dirichlet problem are twice differentiable in the classical sense. In case of the Laplace operator, it is well known that Dini continuity of $f: D \mapsto \mathbb{R}$, i.e. finiteness of the integral $\int_{0}^{1} \omega_{f}(r) / r \mathrm{~d} r$ for the modulus of continuity $\omega_{f}$, implies that the distributional solution $u$ to the classical Dirichlet problem satisfies $u \in C^{2}(D)$. On the other hand, one can construct a continuous function $f: B_{1} \mapsto \mathbb{R}$ and a distribution $u \in \mathcal{D}^{\prime}\left(B_{1}\right)$ such that $\Delta u=f$ in the distributional sense, but $u \notin C^{2}\left(B_{1}\right)$. These observations have been made long time ago [26]. They have been extended to non-translation-invariant operators by several authors [12, $33]$ and to nonlinear problems [14, 31]. Note that there are many more related contributions including treatments of partial differential equations on non-smooth domains. In the present work we treat the simple linear case for a general class of nonlocal operators generating isotropic unimodal Lévy processes.

Let us introduce the objects of our study and formulate our main results. Let $v: \mathbb{R}^{d} \backslash$ $\{0\} \mapsto[0, \infty)$ be a function satisfying

$$
\int_{B_{1} \backslash\{0\}} v(h) \mathrm{d} h=\infty \quad \text { and } \quad \int_{\mathbb{R}^{d} \backslash\{0\}}\left(1 \wedge|h|^{2}\right) v(h) \mathrm{d} h<\infty .
$$

The function $v$ induces a measure $v(\mathrm{~d} h)=v(h) \mathrm{d} h$, which is called the Lévy measure. Note that we use the same symbol for the measure as well as for the density. We study operators of the form

$$
\mathscr{L} u(x)=\lim _{\epsilon \rightarrow 0^{+}} \int_{|y|>\epsilon}(u(x+y)-u(x)) v(y) \mathrm{d} y .
$$

This expression is well defined if $u$ is sufficiently regular in the neighbourhood of $x \in$ $\mathbb{R}^{d}$ and satisfies some integrability condition at infinity. We recall that for $\alpha \in(0,2)$ and $v(\mathrm{~d} h)=c_{d, \alpha}|h|^{-d-\alpha} \mathrm{d} h$ with some appropriate constant $c_{d, \alpha}$, the operator $\mathscr{L}$ equals the fractional Laplace operator $-(-\Delta)^{\alpha / 2}$ on $C_{b}^{2}\left(\mathbb{R}^{d}\right)$. The regularity theory of such operators has been intensively studied recently. For instance, it is well known $[3,18,36-38]$ that the solution of $-(-\Delta)^{\alpha / 2} u=f$ with $f \in C^{\beta}$ belongs to $C^{\alpha+\beta}$ provided that neither $\beta$ nor $\alpha+\beta$ is an integer. A similar result in more general setting is derived in [2].

We adopt a common convention and identify the Lévy measure with its radial profile, i.e. $v(x)=v(|x|), x \in \mathbb{R}^{d}$. Our standing assumption is that $h \mapsto v(h)$ is a non-zero, nonincreasing radial function and that there exists a Lévy measure $v^{*}$ resp. a density $v^{*}$ such that $v^{*}(r)=v(r)$ for $r<r_{0}, v(r) \leqslant v^{*}(r)$ for $r \geqslant r_{0}$ and

$$
\nu^{*}(r) \leqslant C v^{*}(r+1), \quad r \geqslant r_{0},
$$

for some $C \geqslant 1$ and $r_{0}>0$. We remark that without loss of generality we may and do assume that $r_{0}$ is such that the function $(0, \infty) \ni x \mapsto 1 \wedge \nu^{*}(x)$ is monotone. Given an open set $D \subset \mathbb{R}^{d}$, denote by $\mathcal{L}^{1}(D)$ the vector space of all Borel functions $u \in L_{\text {loc }}^{1}$ satisfying

$$
\int_{D}|u(x)|\left(1 \wedge v^{*}(x)\right) \mathrm{d} x<\infty .
$$


The condition $u \in \mathcal{L}^{1}\left(\mathbb{R}^{d}\right)$ is the integrability condition needed to ensure well-posedness in the definition of $\mathscr{L} u$ in distributional sense. Note that the shift of $r_{0}$ results in a different constant in the integral above but does not affect its finiteness. Given an open set, we denote by $G_{D}$ resp. $P_{D}$ the usual Green resp. the Poisson operator associated with $\mathscr{L}$, cf. Section 2. For a definition of the mean-value property and the Kato class $\mathcal{K}$ and $\mathcal{K}(D)$ see Definition 2.1 and Definition 2.3 below. Here is our first result.

Theorem 1.1 Let $D$ be a bounded open set. Suppose that supp $f \subset \bar{D}$, supp $g \subset D^{c}$, $f \in L^{1}(D)$ and $g \in \mathcal{L}^{1}\left(D^{c}\right)$. Let $u \in \mathcal{L}^{1}\left(\mathbb{R}^{d}\right)$ be any distributional solution of the Dirichlet problem

$$
\begin{aligned}
\mathscr{L} u & =f \text { in } D, \\
u & =g \text { in } D^{c} .
\end{aligned}
$$

Then $u(x)+G_{D}[f](x)$ satisfies the mean-value property inside D. Furthermore, if $D$ is a Lipschitz domain and there exists $V \subset \subset D$ such that $f$ and $g * v$ belong to the Kato class $\mathcal{K}(D \backslash \bar{V})$, then

$$
u(x)=-G_{D}[f](x)+P_{D}[g](x)
$$

is a unique solution among all solutions which are bounded close to the boundary of $D$.

The theorem above says that the distributional solution of Eq. 1.4 is unique up to a function with the mean-value property. If, additionally, $D$ is a Lipschitz domain and we impose some regularity, then there is a unique solution among all solutions which are bounded close to the boundary. Observe that we do not assume boundedness of $g$, so, in general, $u$ may be unbounded outside of $D$, even close to the boundary. Here bounded close to the boundary means that the restriction of $u$ to the set $D$ is bounded close to the boundary. Boundedness of $u, f, g$ would suffice, of course. We highlight that in general, there are functions with the mean-value property which are unbounded near to the boundary (see e.g. [6, Lemma 6]), thus, in order to address the uniqueness problem, we need to restrict ourselves to solutions bounded close to the boundary. Observe that in the first part of the theorem we do not say anything about existence of solutions; we are able to establish it only after additional assumptions on $f$ and $g$. A more expanded discussion on the existence of solutions for problems driven by a non-local operator can be found in [8]. Note that, in the case where $\mathscr{L}$ equals the fractional Laplace operator, similar results like Theorem 1.1 are proved in [7]. A result similar to Theorem 1.1 has recently been proved in [29]. The authors consider a smaller class of operators and concentrate on viscosity solutions instead of distributional ones.

Variational solutions to nonlocal operators have been studied by several authors, e.g., in $[17,40]$. The problem to determine appropriate function spaces for the data $g$ leads to the notion of nonlocal traces spaces introduced in [15]. It is interesting that the study of Dirichlet problems for nonlocal operators leads to new questions regarding the theory of function spaces.

The formulation of our second main result requires some further preparation. They are rather technical because we cover a large class of translation-invariant operators. The similar condition to the following appears in [8].

(A) $\quad v$ is twice continuously differentiable and there is a positive constant $C$ such that

$$
\left|v^{\prime}(r)\right|,\left|\nu^{\prime \prime}(r)\right| \leqslant C \nu^{*}(r) \quad \text { for } r \geqslant r_{0} .
$$


(A) and Eq. 1.2 are essential for proving that functions with the mean-value property are twice continuously differentiable, see Lemma 2.2. We emphasize that in general this is not the case and usually such functions lack sufficient regularity if no additional assumptions are imposed. The reader is referred to [32, Example 7.5], where a function $f$ with the mean-value property is constructed for which $f^{\prime}(0)$ does not exist.

Let $G$ be a minus fundamental solution of $\mathscr{L}$ on $\mathbb{R}^{d}$ (see Eq. 2.2 for definition). Note that in the case of the fractional Laplace operator $G(x)=c_{d, \alpha}|x|^{\alpha-d}$ for $d \neq \alpha$ and some constant $c_{d, \alpha}$ (see e.g. [10]). In what follows we will assume the kernel $G$ to satisfy the following growth condition:

(G) $\quad G \in C^{2}\left(\mathbb{R}^{d} \backslash\{0\}\right)$ and there exists a non-increasing function $S:(0, \infty) \mapsto[0, \infty)$ and $r_{0}>0$ such that

(i) if $\int_{0}^{1 / 2}\left|G^{\prime}(t)\right| t^{d-1} \mathrm{~d} t=\infty$, then

$$
G(r),\left|G^{\prime}(r)\right|, r\left|G^{\prime \prime}(r)\right| \leqslant S(r), \quad r<r_{0},
$$

(ii) if $\int_{0}^{1 / 2}\left|G^{\prime}(t)\right| t^{d-1} \mathrm{~d} t<\infty$, then additionally $G \in C^{3}\left(\mathbb{R}^{d} \backslash\{0\}\right)$ and

$$
G(r),\left|G^{\prime}(r)\right|,\left|G^{\prime \prime}(r)\right|, r\left|G^{\prime \prime \prime}(r)\right| \leqslant S(r), \quad r<r_{0} .
$$

Theorem 1.2 Let $D$ be an open bounded set and $f: D \mapsto \mathbb{R}$. Assume that the measure $v$ satisfies (A) and Eq. 1.2 and the fundamental solution $G$ satisfies $(G)$. If $\int_{0}^{1}\left|G^{\prime}(t)\right| t^{d-1} \mathrm{~d} t<$ $\infty$ we assume

$$
\int_{0}^{1 / 2} S(t) \omega_{f}(t, D) t^{d-1} \mathrm{~d} t<\infty
$$

or if $\int_{0}^{1}\left|G^{\prime}(t)\right| t^{d-1} \mathrm{~d} t=\infty$ we assume

$$
\int_{0}^{1 / 2} S(t) \omega_{\nabla f}(t, D) t^{d-1} \mathrm{~d} t<\infty .
$$

Then any distributional solution $u \in \mathcal{L}^{1}\left(\mathbb{R}^{d}\right)$ of the problem

$$
\mathscr{L} u=f \text { in } D
$$

belongs to $C^{2}(D)$ and is unique up to a function with the mean-value property.

Remark 1.3 Equations 1.5 or 1.6 imply $f \in \mathcal{K}(D)$, so by Theorem 1.1 , if $D$ is a bounded Lipschitz domain, $g \in \mathcal{L}^{1}\left(D^{c}\right)$ and $g * v \in \mathcal{K}(D)$ then there exists a unique solution of Eq. 1.4 among all solutions which are bounded close to the boundary.

The result uses quite involved conditions because the measure $v$ interacts with the Dinitype assumptions for the right-hand side function $f$. Looking at examples, we see that the two cases described in the theorem appear naturally. In the fractional Laplacian case $\left(G(x)=c_{d, \alpha}|x|^{\alpha-d}\right.$ for $\left.d \neq \alpha\right)$, finiteness of the expression $\int_{0}^{1 / 2}\left|G^{\prime}(t)\right| t^{d-1} \mathrm{~d} t$ depends on the value of $\alpha \in(0,2)$. We show in Section 6 that the conditions hold true when $\mathscr{L}$ is the generator of a rotationally symmetric $\alpha$-stable process, i.e., when $\mathscr{L}$ equals the fractional Laplace operator. Note that Theorem 1.2 is a new result even in this case. We also study the more general class, e.g. operators of the form $-\varphi(-\Delta)$, where $\varphi$ is a Bernstein function. Note that in the theorems and remark above we do not assume that $g$ is bounded. 
Remark 1.4 We emphasize that in the case of $\mathscr{L}$ being the fractional Laplace operator of order $\alpha \in(0,2)$ and $f \in C^{2-\alpha}\left(B_{1}\right)$, it is not true that the function $x \mapsto$ $\int_{B_{1}} G_{B_{1}}(x, y) f(y) \mathrm{d} y$ belongs to $C^{2}\left(B_{1}\right)$, or even to $C^{1,1}\left(B_{1}\right)$ as is stated in [1, Theorem 3.7]. A similar phenomenon has been mentioned in [3] and is visible here as well. Observe that in such case the integrals (1.5) and (1.6) are clearly divergent and consequently, Theorem 1.2 cannot be applied. We devote Section 5 to the construction of counterexamples for any $\alpha \in(0,2)$. On the other hand, by [39, Theorem 1.1(b)], those counterexamples are $C^{1,1-\epsilon}\left(B_{1}\right)$ for every $\epsilon \in(0,1)$ and consequently, they are also pointwise solutions. Thus, $[1$, Theorem 1.1] is also not true in general.

The article is organized as follows: in Section 2 we provide the main definitions and some preliminary results. The proof of Theorem 1.1 is provided in Section 3. Section 4 contains several rather technical computations and the proof of Theorem 1.2. We discuss the necessity of the assumptions of Theorem 1.2 through examples in Section 5. Finally, in Section 6 we provide examples that show that the assumptions of Theorem 1.2 are natural.

\section{Preliminaries}

In this section we explain our use of notation, define several objects and collect some basic facts. We write $f \asymp g$ when $f$ and $g$ are comparable, that is the quotient $f / g$ stays between two positive constants. To simplify the notation, for a radial function $f$ we use the same symbol to denote its radial profile. In the whole paper $c$ and $C$ denote constants which may vary from line to line. We write $c_{a}$ when the constant $c$ depends only on $a$. By $B(x, r)$ we denote the ball of radius $r$ centered at $x$, that is $B(x, r)=\left\{y \in \mathbb{R}^{d}:|y-x|<r\right\}$. For convenience we set $B_{r}=B(0, r)$. For an open set $D$ and $x \in D$ we define $\delta_{D}(x)=$ $\operatorname{dist}(x, \partial D)$ and $\operatorname{diam}(D)=\sup _{x, y \in D}|x-y|$. The modulus of continuity of a continuous function $f: D \mapsto \mathbb{R}$ is defined by

$$
\omega_{f}(t, D)=\sup \{|f(x)-f(y)|: x, y \in D,|x-y|<t\}, \quad t>0 .
$$

For a differentiable function $f: D \mapsto \mathbb{R}$ we set

$$
\omega_{\nabla f}(t, D)=\max _{i \in\{1, \ldots, d\}} \sup \left\{\left|\partial_{x_{i}} f(x)-\partial_{x_{i}} f(y)\right|: x, y \in D,|x-y|<t\right\}, \quad t>0 .
$$

We say that a Borel measure is isotropic unimodal if it is absolutely continuous on $\mathbb{R}^{d} \backslash\{0\}$ with respect to the Lebesgue measure and has a radial, non-increasing density. Given an isotropic unimodal Lévy measure $v(\mathrm{~d} x)=v(|x|) \mathrm{d} x$, we define a Lévy-Khinchine exponent

$$
\psi(\xi)=\int_{\mathbb{R}^{d}}(1-\cos (\xi \cdot x)) v(\mathrm{~d} x), \quad \xi \in \mathbb{R}^{d} .
$$

$\psi$ is usually called the characteristic exponent. It is well known (e.g. [32, Lemma 2.5]) that if $v\left(\mathbb{R}^{d}\right)=\infty$, there exists a continuous function $p_{t} \geqslant 0$ in $\mathbb{R}^{d} \backslash\{0\}$ such that

$$
\widehat{p}_{t}(\xi)=\int_{\mathbb{R}^{d}} e^{-i \xi \cdot x} p_{t}(x) \mathrm{d} x=e^{-t \psi(\xi)}, \quad \xi \in \mathbb{R}^{d} .
$$

The family $\left\{p_{t}\right\}_{t>0}$ induces a strongly continuous contraction semigroup on $C_{0}\left(\mathbb{R}^{d}\right)$ and $L^{2}\left(\mathbb{R}^{d}\right)$

$$
P_{t} f(x)=\int_{\mathbb{R}^{d}} f(y) p_{t}(y-x) \mathrm{d} y, \quad x \in \mathbb{R}^{d},
$$


whose generator $\mathcal{A}$ has the Fourier symbol $-\psi$. Using the Kolmogorov theorem one can construct a stochastic process $X_{t}$ with transition densities $p_{t}(x, y)=p_{t}(y-x)$, namely $\mathbb{P}^{x}\left(X_{t} \in A\right)=\int_{A} p_{t}(x, y) \mathrm{d} y$. Here $\mathbb{P}^{x}$ is the probability corresponding to a process $X_{t}$ starting from $x$, that is $\mathbb{P}^{x}\left(X_{0}=x\right)=1$. By $\mathbb{E}^{x}$ we denote the corresponding expectation. In fact, $X_{t}$ is a pure-jump isotropic unimodal Lévy process in $\mathbb{R}^{d}$, that is a stochastic process with stationary and independent increments and càdlàg paths, whose transition function is absolutely continuous and its density $p_{t}(x)$ is isotropic unimodal, that is radial and radially non-increasing (see for instance [41] and [44]).

One of the objects of significant importance in this paper is the potential kernel defined as follows:

$$
U(x, y)=\int_{0}^{\infty} p_{t}(x, y) \mathrm{d} t .
$$

Clearly $U(x, y)=U(y-x)$. The potential kernel can be defined in our setting if $\int_{B_{1}} \frac{1}{\psi(\xi)} d \xi<\infty$. In particular, for $d \geqslant 3$ the potential kernel always exists (see [41, Theorem 37.8]). If this is not the case, one can consider the compensated potential kernel

$$
W_{x_{0}}(x-y)=\int_{0}^{\infty}\left(p_{t}(x-y)-p_{t}\left(x_{0}\right)\right) \mathrm{d} t
$$

for some fixed $x_{0} \in \mathbb{R}^{d}$. If $d=1, \int_{B_{1}} \frac{\mathrm{d} \xi}{\psi(\xi)}=\infty$ and $\int_{\mathbb{R}} \frac{\mathrm{d} \xi}{1+\psi(\xi)}<\infty$, we can set $x_{0}=0$. In other cases the compensation must be taken with $x_{0} \in \mathbb{R}^{d} \backslash\{0\}$. For details we refer the reader to [21] and to Appendix A.

Slightly abusing the notation, we let $W_{1}$ be Eq. 2.1 for $x_{0}=(0, \ldots, 0,1) \in \mathbb{R}^{d}$. Thus, we have arrived with three potential kernels: $U, W_{0}$ and $W_{1}$. Each one corresponds to a different type of process $X_{t}$ and an operator associated with it. In order to merge these cases in one object, we let

$$
G(x)=\left\{\begin{array}{l}
U(x), \text { if } \int_{B_{1}} \frac{\mathrm{d} \xi}{\psi(\xi)}<\infty \\
W_{0}(x), \text { if } d=1, \int_{B_{1}} \frac{\mathrm{d} \xi}{\psi(\xi)}=\infty \text { and } \int_{0}^{\infty} \frac{1}{1+\psi(\xi)} \mathrm{d} \xi<\infty, \\
W_{1}(x), \text { otherwise. }
\end{array}\right.
$$

For instance, in the case of $\mathscr{L}=\Delta$ we have

$$
G(x)= \begin{cases}c_{d}|x|^{2-d}, & d \geqslant 3, \\ \frac{1}{2 \pi} \ln \frac{1}{|x|}, & d=2, \\ -\frac{1}{2}|x|, & d=1 .\end{cases}
$$

The basic object in the theory of stochastic processes is the first exit time of $X$ from $D$,

$$
\tau_{D}=\inf \left\{t>0: X_{t} \notin D\right\} .
$$

Using $\tau_{D}$ we define an analogue of the generator of $X_{t}$, namely, the characteristic operator or Dynkin operator. We say a Borel function $f$ is in a domain $\mathcal{D}_{\mathcal{U}}$ of Dynkin operator $\mathcal{U}$ if there exists a limit

$$
\mathcal{U} f(x)=\lim _{B \rightarrow\{x\}} \frac{\mathbb{E}^{x} f\left(X_{\tau_{B}}\right)-f(x)}{\mathbb{E}^{x} \tau_{B}} .
$$

Here $B \rightarrow\{x\}$ is understood as a limit over all sequences of open sets $B_{n}$ whose intersection is $\{x\}$ and whose diameters tend to 0 as $n \rightarrow \infty$. The characteristic operator is an extension of $\mathcal{A}$ on $L^{\infty}$, that is $\mathcal{D}_{\mathcal{A}} \subset \mathcal{D}_{\mathcal{U}}$ and $\left.\mathcal{U}\right|_{\mathcal{D}_{\mathcal{A}}}=\mathcal{A}$. For a wide description of characteristic operator and its relation with the generator of $X_{t}$ we refer the reader to [16, Chapter V]. 
Instead of the whole $\mathbb{R}^{d}$, one can consider a process $X$ killed after exiting $D$. By $p_{t}^{D}(x, y)$ we denote its transition density (or, in other words, the fundamental solution of $\partial_{t}-\mathscr{L}$ in $D)$. We have

$$
p_{t}^{D}(x, y)=p_{t}(x, y)-\mathbb{E}^{x}\left[\tau_{D}<t ; p_{t-\tau_{D}}\left(X_{\tau_{D}}, y\right)\right], \quad x, y \in \mathbb{R}^{d} .
$$

It follows that $0 \leqslant p_{t}^{D} \leqslant p_{t}$. Proceeding as in the proof of [13, Theorem 2.1] one may check that $p_{t}^{D}$ is symmetric. We define the Green function for the set $D$

$$
G_{D}(x, y)=\int_{0}^{\infty} p_{t}^{D}(x, y) \mathrm{d} t, \quad x, y \in \mathbb{R}^{d},
$$

and the Green operator

$$
G_{D}[f](x)=\int_{D} G_{D}(x, y) f(y) \mathrm{d} y, \quad x \in \mathbb{R}^{d},
$$

for suitable functions $f$. Since $p_{t}^{D}$ is symmetric, it follows that $G_{D}$ is also symmetric. We note that $G_{D}(x, y)$ can be interpreted as the occupation time density up to the exit time $\tau_{D}$. Using that we obtain $G_{D}[\mathbf{1}](x)=\mathbb{E}^{x} \tau_{D}$. For bounded sets $D$ we have $\sup _{x \in \mathbb{R}^{d}} \mathbb{E}^{x} \tau_{D}<\infty$ $[9,35]$. By the strong Markov property, for any open $\Omega \subset D$ we have

$$
G_{D}(x \cdot y)=G_{\Omega}(x, y)+\mathbb{E}^{x} G_{D}\left(X_{\tau_{\Omega}}, y\right), \quad x, y \in \mathbb{R}^{d} .
$$

Obviously we have $G_{\mathbb{R}^{d}}=U$. If $U$ is well-defined (finite) a.e., the well-known Hunt formula holds:

$$
G_{D}(x, y)=U(y-x)-\mathbb{E}^{x} U\left(y-X_{\tau_{D}}\right), \quad x, y \in \mathbb{R}^{d} .
$$

In case of compensated potential kernels, a similar formula is valid, namely,

$$
G_{D}(x, y)=G(y-x)-\mathbb{E}^{x} G\left(y-X_{\tau_{D}}\right), \quad x, y \in \mathbb{R}^{d} .
$$

See Theorem A.4.

By $P_{D}(x, \mathrm{~d} z)$ we denote the distribution of $X_{\tau_{D}}$ with respect to $\mathbb{P}^{x}$, that is $P_{D}(x, A)=$ $\mathbb{P}^{x}\left(X_{\tau_{D}} \in A\right)$. We call $P_{D}(x, \mathrm{~d} z)$ the harmonic measure and its density $P_{D}(x, z)$ on $\bar{D}^{c}$ with respect to the Lebesgue measure - the Poisson kernel. The Ikeda-Watanabe formula [27] states that

$$
P_{D}(x, z)=\int_{D} G_{D}(x, y) v(z-y) \mathrm{d} y, \quad x \in D, z \in \bar{D}^{c} .
$$

Recall that for $D$ being the Lipschitz set, $P_{D}(x, \mathrm{~d} z)$ does not contain a singular part on $\partial D$, i.e. we have $P_{D}(x, \partial D)=0$ for all $x \in D$, see e.g. [43]. For $g: D^{c} \mapsto \mathbb{R}$ we let

$$
P_{D}[g](x)=\int_{D^{c}} g(z) P_{D}(x, \mathrm{~d} z), \quad x \in D,
$$

if the integral exists. For $x \in D^{c}$ we set $P_{D}[g](x)=g(x)$.

Definition 2.1 We say that a function $g: \mathbb{R}^{d} \mapsto \mathbb{R}$ satisfies the mean-value property in an open set $D \subset \mathbb{R}^{d}$ if $g(x)=P_{D}[g](x)$ for all $x \in D$. Here we assume that the integral is absolutely convergent. If $g$ has the mean-value property in every bounded open set whose closure is contained in $D$ then $g$ is said to have the mean-value property inside $D$.

Clearly if $f$ has the mean-value property inside $D$, then $\mathcal{U} f=0$ in $D$.

In general, functions with the mean-value property lack sufficient regularity if no additional assumptions are imposed. In our setting, however, we can show that they are, in fact, twice continuously differentiable in $D$. 
Lemma 2.2 Let $u \in \mathcal{L}^{1}$ and $D$ be an open set. Suppose that (A) and Eq. 1.2 hold. If $u$ has the mean-value property inside $D$, then $u \in C^{2}(D)$.

The proof is similar to the proof of [8, Theorem 4.6] and is omitted.

Definition 2.3 $[25,45]$ We say that a Borel function $f$ belongs to the Kato class $\mathcal{K}$ if it satisfies the following condition

$$
\lim _{r \rightarrow 0^{+}}\left[\sup _{x \in \mathbb{R}^{d}} \int_{0}^{r} P_{t}|f|(x) \mathrm{d} t\right]=0 .
$$

We say that $f \in \mathcal{K}(D)$, where $D$ is an open set, if $f \mathbf{1}_{D} \in \mathcal{K}$.

This is one of three conditions discussed by Zhao in [45]. A detailed description of different notions of the Kato class and related conditions can be found in [25].

Lemma 2.4 Let $V \subset \subset D$ and $\rho:=\operatorname{dist}(V, \partial D)$. Suppose $f \in L^{1}(D) \cap \mathcal{K}(D \backslash \bar{V})$. Then $G_{D}[f]$ is bounded in $V_{1}:=\left\{x \in D \backslash V: \delta_{D}(x)<\rho / 2\right\}$.

Proof Let $x \in V_{1}$ and define $V_{2}:=\left\{x \in D \backslash \bar{V}: \delta_{D}(x)<3 \rho / 4\right\}$. We have

$$
\left|G_{D}\left[f \mathbf{1}_{V_{2}^{c}}\right](x)\right| \leqslant \int_{V_{2}^{c}} G_{D}(x, y)|f(y)| \mathrm{d} y .
$$

Let $r=2 \sup _{x \in D}|x|$. Then $D \subset B_{r}$ and by [20, Theorem 1.3],

$$
\int_{V_{2}^{c}} G_{D}(x, y)|f(y)| \mathrm{d} y \leqslant \int_{V_{2}^{c}} G_{B_{r}}(x, y)|f(y)| \mathrm{d} y \leqslant c_{r, \rho}\|f\|_{L^{1}(D)} .
$$

Moreover, by Eq. 2.3,

$$
G_{D}\left[f \mathbf{1}_{V_{2}}\right](x)=G_{D \backslash \bar{V}}\left[f \mathbf{1}_{V_{2}}\right](x)+\mathbb{E}^{x} G_{D}\left[f \mathbf{1}_{V_{2}}\right]\left(X_{\tau_{D \backslash \bar{V}}}\right) .
$$

Observe that

$$
\left|\mathbb{E}^{x} G_{D}\left[f \mathbf{1}_{V_{2}}\right]\left(X_{\tau_{D \backslash \bar{V}}}\right)\right| \leqslant \mathbb{E}^{x} \int_{V_{2}} G_{D}\left(X_{\tau_{D \backslash \bar{V}}}, y\right)|f(y)| \mathrm{d} y \leqslant c_{\rho}\|f\|_{L^{1}(D)},
$$

again by [20, Theorem 1.3]. Finally, we have

$$
\left|G_{D \backslash \bar{V}}\left[f \mathbf{1}_{V_{2}}\right](x)\right| \leqslant G_{D \backslash \bar{V}}\left[|f| \mathbf{1}_{D \backslash \bar{V}}\right](x) .
$$

A straightforward application of the proof of [13, Theorem 4.3(i)] to the last term yields the claim.

Proposition 2.5 Assume $(G)$. If $f$ satisfies (1.5) then it is uniformly continuous in $D$. If Eq. 1.6 holds then $\frac{\partial}{\partial x_{i}} f, i=1, \ldots, d$, are uniformly continuous in $D$.

Proof Suppose $\frac{\partial}{\partial x_{i}} f$ for some $i=1, \ldots, d$ is not uniformly continuous, i.e. $\omega_{\nabla f}(t, D) \geqslant$ $c>0$ for every $t>0$. If Eq. 1.6 holds then in particular

$$
\infty>\int_{0}^{1 / 2} S(t) \omega_{\nabla f}(t, D) t^{d-1} \mathrm{~d} t \geqslant c \int_{0}^{1 / 2}\left|G^{\prime}(t)\right| t^{d-1} \mathrm{~d} t
$$


which is a contradiction. Now let $\omega_{f}(t, D) \geqslant c$ for $t>0$, and suppose Eq. 1.5. We have

$$
\infty>\int_{0}^{1 / 2} S(t) \omega_{f}(t, D) t^{d-1} \mathrm{~d} t \geqslant c \int_{0}^{1 / 2}\left|G^{\prime \prime}(t)\right| t^{d-1} \mathrm{~d} t
$$

Let us notice that by monotonicity of $p_{t}$ and definition of $G$, its derivative $G^{\prime}$ is of constant sign on $(0,1)$. By integration by parts,

$$
\int_{0}^{1 / 2} G^{\prime \prime}(t) t^{d-1} \mathrm{~d} t=\left.G^{\prime}(t) t^{d-1}\right|_{0} ^{1 / 2}-(d-1) \int_{0}^{1 / 2} G^{\prime}(t) t^{d-2} \mathrm{~d} t .
$$

First let $d \geqslant 3$. Then it follows that both $\lim _{t \rightarrow 0^{+}} G^{\prime}(t) t^{d-1}$ and the integral are finite. In particular, integration by parts once again yields

$$
\int_{0}^{1 / 2} G^{\prime}(t) t^{d-2} \mathrm{~d} t=\left.G(t) t^{d-2}\right|_{0} ^{1 / 2}-(d-2) \int_{0}^{1 / 2} G(t) t^{d-3} \mathrm{~d} t .
$$

Both $\lim _{t \rightarrow 0^{+}} G(t) t^{d-2}$ and the integral are positive. Hence, both must be finite. By [19, Proposition 1 and 2] we have $\int_{0}^{r} G(t) t^{d-1} d t \geqslant c \psi(1 / r)^{-1}, r>0$. Furthermore, for any $d \geqslant 1$ the dominated convergence theorem implies

$$
\lim _{r \rightarrow \infty} \frac{\psi(r)}{r^{2}}=0
$$

Hence,

$$
\begin{gathered}
\int_{0}^{1} G(t) t^{d-3} d t=c_{d} \int_{B_{1}} \frac{G(|x|)}{|x|^{2}} \mathrm{~d} x=c_{d} \int_{B_{1}} \int_{|x|}^{\infty} \frac{1}{s^{3}} \mathrm{~d} s G(|x|) \mathrm{d} x=\frac{c_{d}}{2} \int_{0}^{\infty} \mathrm{d} s \frac{1}{s^{3}} \int_{B_{1 \wedge s}} G(|x|) \mathrm{d} x \\
\geqslant c\left(\int_{0}^{1} \frac{1}{\psi(1 / s) s^{2}} \frac{\mathrm{d} s}{s}+\psi(1)\right) \geqslant c \int_{1}^{\infty} \frac{u^{2}}{\psi(u)} \frac{\mathrm{d} u}{u} \geqslant c \int_{1}^{\infty} \frac{\mathrm{d} u}{u}=\infty,
\end{gathered}
$$

which is a contradiction. Now let $d=2$. By the same argument

$$
\int_{0}^{1 / 2} G^{\prime \prime}(t) t \mathrm{~d} t=\left.G^{\prime}(t) t\right|_{0} ^{1 / 2}-\int_{0}^{1 / 2} G^{\prime}(t) \mathrm{d} t,
$$

and we conclude that the integral is finite. Hence, $\lim _{t \rightarrow 0^{+}} G(t)<\infty$. By [4, Corollary 17 of Section 2] one point sets are essentially polar. Therefore, [4, Theorem 16(iii) of Section 2] and radial monotonicity of $G$ imply that $G$ is unbounded near zero. Indeed, if $G=U$, this is a direct consequence of this theorem. If $G=W_{1}$ then for $x \in B_{1}$ we have $G(|x|)>0$ and consequently,

$$
\int_{0}^{\infty} e^{-t} p_{t}(x) \mathrm{d} t=\int_{0}^{\infty} e^{-t}\left(p_{t}(x)-p_{t}(1)\right) \mathrm{d} t+\int_{0}^{\infty} e^{-t} p_{t}(1) \mathrm{d} t \leqslant G(|x|)+\int_{0}^{\infty} e^{-t} p_{t}(1) \mathrm{d} t,
$$

and by [22, Theorem 5.4], $\int_{0}^{\infty} e^{-t} p_{t}(1) \mathrm{d} t$ is finite. In view of [4, Theorem 16(iii) of Section 2] we get the contradiction. Finally, for $d=1$ we get that $\lim _{t \rightarrow 0^{+}}\left|G^{\prime}(t)\right|<\infty$. It follows that $\limsup _{t \rightarrow 0^{+}}|G(t)| / t<\infty$. Observe that such condition implies that we cannot have $G=U$. Indeed, if $G=U$ then by radial monotonicity of $U$ we would have $U \equiv 0$, which in turn would imply $p_{t} \equiv 0$ for some $t>0$, which is obviously a contradiction. Moreover, similar arguments like in the above inequality imply that the resolvent kernel is bounded. [4, Theorem 16(ii) of Section 2] implies that $G=W_{0}$. Note that by [19, Lemma 1 and Proposition 1], $\psi$ is almost increasing and has a doubling property. Thus, due to [21, Lemma 2.14] we obtain that

$$
\frac{r}{\psi(r)} \leqslant c \int_{r}^{2 r} \frac{d s}{\psi(s)} \leqslant c \int_{r}^{\infty} \frac{d s}{\psi(s)} \leqslant c|G(1 / r)| .
$$


That in turn implies

$$
\liminf _{r \rightarrow \infty} \psi(r) / r^{2}>0
$$

which is a contradiction with Eq. 2.6.

Lemma 2.6 Let $D$ be bounded open and $k \in \mathbb{N}$. If $g \in C^{k}\left(\mathbb{R}^{d} \backslash\{0\}\right) \cap L_{\text {loc }}^{1}$ and $f \in C^{k}(D)$ has compact support in $\mathbb{R}^{d}$ then $g * f \in C^{k}(D)$.

Proof Fix $x_{0} \in D$. Let $l=\delta_{D}\left(x_{0}\right)$. Let $\chi_{1}, \chi_{2} \in C^{\infty}\left(\mathbb{R}^{d}\right)$ be such that $\mathbf{1}_{B\left(x_{0}, l / 4\right)} \leqslant \chi_{1} \leqslant$ $\mathbf{1}_{B\left(x_{0}, l / 2\right)}$ and $\mathbf{1}_{B_{l / 8}^{c}} \leqslant \chi_{2} \leqslant \mathbf{1}_{B_{l / 16}^{c}}$. Observe that $g * f=g *\left(f \chi_{1}\right)+\left(g \chi_{2}\right) *\left(f\left(1-\chi_{1}\right)\right)$ on $B\left(x_{0}, l / 8\right)$. Since $f \chi_{1}, g \chi_{2} \in C^{k}\left(\mathbb{R}^{d}\right)$, it follows that $g * f \in C^{k}\left(B\left(x_{0}, l / 8\right)\right)$. Since $x_{0}$ was arbitrary, the claim follows by induction.

A consequence of Lemma 2.6 is the following corollary.

Corollary 2.7 Let $D$ be open and bounded and $k \in \mathbb{N}$. If $g \in C^{k}\left(\mathbb{R}^{d} \backslash\{0\}\right) \cap L_{\text {loc }}^{1}$ then $g * \mathbf{1}_{D} \in C^{k}(D)$

The following lemma is crucial in one of the proofs.

Lemma 2.8 ([20, Proposition 3.2]) Let $X_{t}$ be an isotropic unimodal Lévy process in $\mathbb{R}^{d}$. For every $r>0$ there is a radial kernel function $\bar{P}_{r}(z)$ and a constant $C(r)>0$ such that $\bar{P}_{r}(z)=C(r)$ for $z \in B_{r}, 0 \leqslant \bar{P}_{r}(z) \leqslant C(r)$ for $z \in \mathbb{R}^{d}$, the profile function of $\bar{P}_{r}$ is non-increasing, and $\bar{P}_{r}(z) \leqslant P_{B_{r}}(0, z)$ for $z \in B_{r}^{c}$. Furthermore, if $f$ has the mean-value property in $B(x, r)$ then

$$
f(x)=\int_{\mathbb{R}^{d}} f(z) \bar{P}_{r}(x-z) \mathrm{d} z=f * \bar{P}_{r}(x) .
$$

Before embarking on our main results let us note one more useful observation.

Lemma 2.9 Suppose $f \in \mathcal{L}^{1}$ and let $\phi_{\epsilon}, \epsilon>0$, be a standard mollifier (i.e. $\phi_{\epsilon} \in C^{\infty}\left(\mathbb{R}^{d}\right)$, $\operatorname{supp} \phi_{\epsilon}=\bar{B}_{\epsilon}$ and $\left.\int_{B_{\epsilon}} \phi_{\epsilon}(x) \mathrm{d} x=1\right)$. Then $f * \phi_{\epsilon} \in \mathcal{L}^{1}$ for any $\epsilon>0$ and $\lim _{\epsilon \rightarrow 0^{+}} f * \phi_{\epsilon}=f$ in $\mathcal{L}^{1}$.

Proof Observe that thanks to Eq. 1.2 we get that $f * \phi_{\epsilon} \in \mathcal{L}^{1}$ for any $\epsilon>0$. Now one may proceed exactly as in the proof of convergence of convolution in the classical $L^{1}$ space.

Remark 2.10 Note that Lemma 2.9 fails without the assumption of doubling condition on $v^{*}$. In fact, this condition is crucial even for the well-posedness of the problem, let alone further results.

\section{Weak Solutions}

The aim of this section is to prove Theorem 1.1. For the fractional Laplacian related results are known, cf. [7, Section 3]. A similar result has recently been obtained in [11] using purely 
analytic methods instead of probabilistic ones exploited in [7]. When the generalization of these results to more general nonlocal operators is immediate, we omit the proof.

Lemma 3.1 Suppose $u \in \mathcal{L}^{1}\left(\mathbb{R}^{d}\right)$ has the mean-value property inside $D$. Then $\mathscr{L} u(x)=0$ for $x \in D$.

Proof First observe that by Lemma 2.2 we have $u \in C^{2}(D)$ and consequently, $\mathscr{L} u(x)$ can be calculated pointwise. Now we proceed exactly as in the proof of [8, Lemma 4.10]. Note that we do not need the assumption A1 from [8], because it is used only to show that the considered function belongs to $C^{2}(D)$; furthermore, since the Lévy density $v(r)$ is nonincreasing, it is continuous in a.e. $r \in(0, \infty)$, so indeed the proof is exactly the same. Thus, the claim follows immediately.

The following lemma is a generalization of [7, Theorem 3.9 and Corollary 3.10], where the fractional Laplace operator is considered.

Lemma 3.2 Let $u \in \mathcal{L}^{1}\left(\mathbb{R}^{d}\right) \cap C^{2}(D)$ be a solution of $\mathscr{L} u=0$ in $D$ in distributional sense. Then $u$ has the mean-value property inside $D$.

Proof Since $u \in \mathcal{L}^{1}\left(\mathbb{R}^{d}\right) \cap C^{2}(D), \mathscr{L} u(x)$ can be calculated pointwise for $x \in D$ and consequently, $\mathscr{L} u(x)=0$ for $x \in D$. Fix $D_{1} \subset \subset D$ and define $\widetilde{u}(x)=P_{D_{1}}[u](x), x \in \mathbb{R}^{d}$. By the strong Markov property we may assume that $D_{1}$ is a Lipschitz domain. We claim that $\tilde{u}$ has the mean-value property in $D_{1}$. Indeed, let $D_{2}$ be an open set relatively compact in $D$ such that $\overline{D_{1}} \subset D_{2}$. There exist functions $u_{1}, u_{2}$ on $D_{1}^{c}$ such that $u=u_{1}+u_{2}, u_{1}$ is continuous and bounded on $D_{1}^{c}$ and $u_{2} \equiv 0$ in $D_{2}$. We have

$$
\tilde{u}(x)=P_{D_{1}}\left[u_{1}\right](x)+P_{D_{1}}\left[u_{2}\right](x), \quad x \in \mathbb{R}^{d} .
$$

The first integral is clearly absolutely convergent. We claim that it is also continuous as a function of $x$ in $\overline{D_{1}}$. Indeed, by Lemma 2.2 it is continuous in $D_{1}$. Let $x_{0} \in \partial D$. For $\epsilon>0$ there exists $\delta>0$ such that

$$
\left|\int_{D_{1}^{c}} P_{D_{1}}(x, z) u_{1}(z) \mathrm{d} z-u_{1}\left(x_{0}\right)\right| \leqslant \epsilon+2\left\|u_{1}\right\|_{\infty} \mathbb{P}^{x}\left(\left|X_{\tau_{D_{1}}}-x_{0}\right|>\delta\right) .
$$

Since the second term goes to 0 as $x \rightarrow x_{0}$ (see [9, Lemmas 2.1 and 2.9]), by arbitrary choice of $\epsilon$ we get the claim.

Furthermore, from monotonicity of $1 \wedge v^{*}(h)$ and the Ikeda-Watanabe formula we obtain

$$
P_{D_{1}}(x, z) \leqslant\left(1 \wedge v^{*}\left(\operatorname{dist}\left(z, D_{1}\right)\right)\right) \mathbb{E}^{x} \tau_{D_{1}}, \quad x \in D_{1}, z \in D_{2}^{c} .
$$

Since $u \in \mathcal{L}^{1}\left(\mathbb{R}^{d}\right)$, Eq. 1.3 implies the absolute convergence of the second integral. Since by [9, Lemma 2.9], $\mathbb{E}^{x} \tau_{D_{1}} \in C_{0}\left(D_{1}\right)$, it is continuous as well. Hence, $\tilde{u}$ is continuous and has the mean-value property in $D_{1}$. Note that $\tilde{u}=u$ on $D_{1}^{c}$, since $D_{1}$ is a Lipschitz domain.

Let $h=\tilde{u}-u$. We now verify that $h \equiv 0$ so that $u=\tilde{u}$ has the mean-value property in $D_{1}$. Using Lemma 3.1 we conclude that $\mathscr{L} \tilde{u}(x)=0$ for $x \in D_{1}$ and consequently, $\mathscr{L} h(x)=0$ for $x \in D_{1}$. Observe that $h$ is continuous and compactly supported. Suppose $x_{0} \in D_{1}$ is such that $h\left(x_{0}\right)=\sup _{x \in D_{1}} h(x)>0$, then

$$
0=\mathscr{L} h\left(x_{0}\right)=\int_{\mathbb{R}^{d}}\left(h(y)-h\left(x_{0}\right)\right) v\left(x_{0}-y\right) \mathrm{d} y,
$$


which implies that $h$ is constant on $\operatorname{supp}(v)+x_{0}$. If $D_{1} \subset \operatorname{supp}(v)+x_{0}$ we get that $h>0$ on $D_{1}$, which is a contradiction due to continuity of $h$ and the fact that $h \equiv 0$ on $\partial D_{1}$. Thus, $h \leqslant 0$. If this is not the case, then we can use the chain rule to get for any $n \in \mathbb{N}$ that $h$ is constant on $n$ supp $(v)+x_{0}$ and eventually get a contradiction. Similarly, $h$ must be non-negative.

Lemma 3.3 Let $u \in \mathcal{L}^{1}\left(\mathbb{R}^{d}\right)$ be a solution of $\mathscr{L} u=0$ in $D$ in distributional sense. Then $u$ has the mean-value property inside $D$.

Proof Let $\Omega \subset \subset D$ be a bounded Lipschitz domain. By [43] and the Ikeda-Watanabe formula we have that the harmonic measure $P_{\Omega}(x, \mathrm{~d} z)$ is absolutely continuous with respect to the Lebesgue measure. Define $\rho=\left(1 \wedge \operatorname{dist}\left(\Omega, D^{c}\right)\right) / 2$ and let $V=\Omega+B_{\rho}$. For $\epsilon<\rho / 4$ we consider standard mollifiers $\phi_{\epsilon}$. Since $\mathscr{L}$ is translation-invariant we have that $\mathscr{L}\left(\phi_{\epsilon} * u\right)=\mathscr{L} u * \phi_{\epsilon}=0$ in $V_{\epsilon}=\left\{x \in D: \operatorname{dist}\left(x, V^{c}\right)>\epsilon\right\}$ in distributional sense. By Lemma 3.2 we obtain

$$
\phi_{\epsilon} * u(x)=P_{\Omega}\left[\phi_{\epsilon} * u\right](x), \quad x \in \Omega .
$$

Note $u \in L_{\text {loc }}^{1}$ implies $\phi_{\epsilon} * u \rightarrow u$ as $\epsilon \rightarrow 0^{+}$in $L_{\text {loc }}^{1}$. Hence, up to the subsequence,

$$
\lim _{\epsilon \rightarrow 0^{+}} \phi_{\epsilon} * u(x)=u(x) \quad \text { a.e. }
$$

Moreover, since $\phi_{\epsilon} * u$ has the mean-value property in $V_{\rho / 2}$, by Lemma 2.8 ,

$$
\phi_{\epsilon} * u(z)=\phi_{\epsilon} * u * \bar{P}_{r}(z), \quad z \in V_{\rho / 2},
$$

for a fixed $0<r<\rho / 4$. Hence, for any $E \subset \Omega^{c}$,

$$
\begin{aligned}
P_{\Omega}\left[\left|\phi_{\epsilon} * u\right| ; V_{\rho / 2} \cap E\right](x) & =\int_{V_{\rho / 2} \cap \Omega^{c} \cap E}\left|\phi_{\epsilon} * u(z)\right| P_{\Omega}(x, z) \mathrm{d} z \\
& =\int_{V_{\rho / 2} \cap \Omega^{c} \cap E}\left|\phi_{\epsilon} * u * \bar{P}_{r}(z)\right| P_{\Omega}(x, z) \mathrm{d} z \\
& \leqslant \int_{B_{\epsilon}} \phi_{\epsilon}(s) \int_{\mathbb{R}^{d}}|u(y)| \int_{V_{\rho / 2} \cap \Omega^{c} \cap E} \bar{P}_{r}(z-y-s) P_{\Omega}(x, z) \mathrm{d} z \mathrm{~d} y \mathrm{~d} s .
\end{aligned}
$$

Let $c=2 \sup _{x \in V}|x|$. Then from boundedness of $\bar{P}_{r}$ and local integrability of $u$ we get

$$
\begin{aligned}
\int_{|y| \leqslant c}|u(y)| \int_{V_{\rho / 2} \cap \Omega^{c} \cap E} \bar{P}_{r}(z-y-s) P_{\Omega}(x, z) \mathrm{d} z \mathrm{~d} y & \leqslant C \int_{|y| \leqslant c}|u(y)| \mathrm{d} y \int_{E} P_{\Omega}(x, z) \mathrm{d} z \\
& \leqslant C\|u\|_{\mathcal{L}^{1}} \int_{E} P_{\Omega}(x, z) \mathrm{d} z .
\end{aligned}
$$

Furthermore, for $|y|>c$ we have $|z-y-s|>r$, hence $\bar{P}_{r}(z-y-s) \leqslant P_{B_{r}}(0, z-y-s)$. From Eq. 1.2, the Ikeda-Watanabe formula and monotonicity of the Lévy measure we get

$$
P_{B_{r}}(0, y+s-z) \leqslant C\left(1 \wedge v^{*}(|y+s-z|-r)\right) \mathbb{E}^{x} \tau_{B_{r}} \leqslant C\left(1 \wedge v^{*}(|y|)\right) .
$$

The constant $C$ depends only on $\rho$. Thus,

$$
\int_{|y|>c}|u(y)| \int_{V_{\rho / 2} \cap \Omega^{c} \cap E} \bar{P}_{r}(z-y-s) P_{\Omega}(x, z) \mathrm{d} z \mathrm{~d} y \leqslant C\|u\|_{\mathcal{L}^{1}} \int_{E} P_{\Omega}(x, z) \mathrm{d} z .
$$


It follows that $\phi_{\epsilon} * u$ are uniformly integrable with respect to the measure $P_{\Omega}(x, z) \mathrm{d} z$ in $V_{\rho / 2}$. By the Vitali convergence theorem,

$$
\lim _{\epsilon \rightarrow 0^{+}} P_{\Omega}\left[\phi_{\epsilon} * u ; V_{\rho / 2}\right](x)=P_{\Omega}\left[u ; V_{\rho / 2}\right](x) .
$$

It remains to show that $\lim _{\epsilon \rightarrow 0^{+}} P_{\Omega}\left[\phi_{\epsilon} * u ; V_{\rho / 2}^{c}\right]=P_{\Omega}\left[u ; V_{\rho / 2}^{c}\right]$. Since $\operatorname{dist}\left(\Omega, V_{\rho / 2}^{c}\right)=$ $\rho / 2$, by the Ikeda-Watanabe formula,

$$
\begin{aligned}
P_{\Omega}\left[\phi_{\epsilon} * u ; V_{\rho / 2}^{c}\right](x) & =\int_{V_{\rho / 2}} \phi_{\epsilon} * u(z) \int_{\Omega} G_{\Omega}(x, y) v(z-y) \mathrm{d} z \mathrm{~d} y \\
& =\int_{B_{\rho / 2}^{c}} v(z) \mathrm{d} z \int_{\Omega} \phi_{\epsilon} * u(z+y) 1_{V_{\rho / 2}^{c}}(z+y) G_{\Omega}(x, y) \mathrm{d} y .
\end{aligned}
$$

Using the fact that $\int_{\Omega} G_{\Omega}(x, y) \mathrm{d} y=\mathbb{E}^{x} \tau_{\Omega}<\infty, v\left(B_{\rho / 2}^{c}\right)<\infty$ and, in view of Lemma $2.9, \lim _{\epsilon \rightarrow 0^{+}} \phi_{\epsilon} * u=u$ in $\mathcal{L}^{1}\left(\mathbb{R}^{d}\right)$, we obtain

$$
\lim _{\epsilon \rightarrow 0^{+}} P_{\Omega}\left[\phi_{\epsilon} * u ; V_{\rho / 2}^{c}\right]=P_{\Omega}\left[u ; V_{\rho / 2}^{c}\right] .
$$

Thus, $u(x)=P_{\Omega}[u](x)$ for a.e. $x \in \Omega$.

Combining Lemma 3.1 and Lemma 3.3 we immediately obtain the following result.

Theorem 3.4 Let $D$ be an open set and $u \in \mathcal{L}^{1}$. Then $u$ has the mean-value property inside $D$ if and only if $\mathscr{L} u=0$ in $D$ in distributional sense.

Lemma 3.5 Let $D$ be a bounded open set and $f \in L^{1}(D)$. Then $-G_{D}[f]$ is a distributional solution of Eq. 1.4 with $g \equiv 0$.

Proof First assume $f$ is continuous. Then by [16, Chapter V] we have

$$
\mathcal{U} G_{D}[f](x)=-f(x), \quad x \in D .
$$

Since $D$ is bounded, we have $\left\|G_{D}[f]\right\|_{L^{1}(D)} \leqslant\left\|G_{D}[1]\right\|_{\infty}\left\|f \mathbf{1}_{D}\right\|_{L^{1}(D)}$ and

$$
\left\|G_{D}[1]\right\|_{\infty}=\sup _{x \in \mathbb{R}^{d}} G_{D}[1](x)=\sup _{x \in \mathbb{R}^{d}} \mathbb{E}^{x} \tau_{D} \leqslant \mathbb{E}^{0} \tau_{B(0, \operatorname{diam}(D))}<\infty .
$$

Let $\phi_{\epsilon}, \epsilon>0$, be a standard mollifier. Since $\mathcal{U}$ is an extension of $\mathscr{L}$ and is translationinvariant we get

$$
\mathscr{L}\left(\phi_{\epsilon} * G_{D}[f]\right)=\mathcal{U}\left(\phi_{\epsilon} * G_{D}[f]\right)=\phi_{\epsilon} * \mathcal{U} G_{D}[f]=-\phi_{\epsilon} * f .
$$

Thus,

$$
\left(-\phi_{\epsilon} * G_{D}[f], \mathscr{L} \varphi\right)=\left(\phi_{\epsilon} * f, \varphi\right), \quad \varphi \in C_{c}^{\infty}\left(\mathbb{R}^{d}\right) .
$$

Thus, passing $\epsilon \rightarrow 0^{+}$we obtain

$$
\left(-G_{D}[f], \mathscr{L} \varphi\right)=(f, \varphi), \quad \varphi \in C_{c}^{\infty}\left(\mathbb{R}^{d}\right) .
$$

In general case, using mollification of $f$ we get the claim.

Proof of Theorem 1.1 Let $h=u+G_{D}[f]$. By Lemma 3.5, $h$ satisfies $\mathscr{L} u=0$ in $D$ in distributional sense. Hence, by Lemma $3.3 h$ has the mean-value property, which finishes the first claim. 
Now let $f, g * v \in \mathcal{K}(D \backslash \bar{V})$ and $D$ be a Lipschitz domain. Then it follows that

$$
\tilde{u}(x)=-G_{D}[f](x)+P_{D}[g](x) .
$$

is a solution of Eq. 1.4, which is bounded near to the boundary. Assume $u$ is another solution of Eq. 1.4 bounded close to the boundary. We will show that $\tilde{u}=u$ a.e. Let $U_{n} \nearrow D$ be a sequence of Lipschitz domains approaching $D$. We have

$$
P_{U_{n}}[h](x)=P_{U_{n}}\left[h ; D^{c}\right](x)+P_{U_{n}}\left[h ; \bar{D} \backslash U_{n}\right](x) .
$$

By the dominated convergence theorem $P_{U_{n}}\left[h ; D^{c}\right](x) \stackrel{n \rightarrow \infty}{\longrightarrow} P_{D}\left[h ; D^{c}\right](x)=$ $P_{D}[g](x)$. Note that by our additional assumptions on $g$ and $v$ we have that $P_{D}[g]$ is welldefined. Furthermore, there exists $n_{0} \in \mathbb{N}$ such that for $n \geqslant n_{0}$ we have $V \subset U_{n}$. From boundedness of $u$ and Lemma 2.4 we get that $h$ is bounded in $\bar{D} \backslash U_{n}$ for $n>n_{0}$ and

$$
P_{U_{n}}\left[h ; \bar{D} \backslash U_{n}\right](x) \leqslant C P_{U_{n}}\left(x, \bar{D} \backslash U_{n}\right) .
$$

By [43, Theorem 1] we have

$$
P_{U_{n}}\left(x, \bar{D} \backslash U_{n}\right) \stackrel{n \rightarrow \infty}{\longrightarrow} P_{D}(x, \partial D)=0
$$

Hence, $u=\tilde{u}$.

\section{The Sufficient Condition for Twice Differentiability}

In this section, we provide auxiliary technical results and the proof of Theorem 1.2. Throughout this section we assume that $D \subset \mathbb{R}^{d}$ is an open bounded set. The following lemmas are modifications of Lemma 2.2 and Lemma 2.3 in [12].

Lemma 4.1 Suppose $f$ is a uniformly continuous function on $D$ and $H(x, y)$ is a continuous function for $x, y \in D, x \neq y$ satisfying

$$
|H(x, y)| \leqslant F(|x-y|), \quad\left|\frac{\partial H(x, y)}{\partial x_{i}}\right| \leqslant \frac{F(|x-y|)}{|x-y|}, \quad i=1, \ldots, d
$$

for some non-increasing function $F:(0, \infty) \mapsto[0, \infty)$. If the following holds

$$
\int_{0}^{1 / 2} F(t) \omega_{f}(t, D) t^{d-1} \mathrm{~d} t<\infty,
$$

then the function $g(x)=\int_{D} H(x, y)(f(y)-f(x))$ dy is uniformly continuous in D.

Remark 4.2 The integral condition (4.1) and boundedness of the integrand for $1 / 2 \leqslant t \leqslant$ $\operatorname{diam}(D)$ imply that

$$
\int_{0}^{\operatorname{diam}(D)} F(t) \omega_{f}(t, D) t^{d-1} \mathrm{~d} t<\infty
$$

Moreover,

$$
\lim _{h \rightarrow 0^{+}} h \int_{h}^{\operatorname{diam}(D)} F(t) \omega_{f}(t, D) t^{d-2} \mathrm{~d} t=0 .
$$

Indeed, clearly we have

$$
h \int_{h}^{\operatorname{diam}(D)} F(t) \omega_{f}(t, D) t^{d-2} \mathrm{~d} t=\int_{0}^{\operatorname{diam}(D)} \mathbf{1}_{[h, \infty)}(t) F(t) \omega_{f}(t, D) t^{d-1} \frac{h}{t} \mathrm{~d} t .
$$


Since $\mathbf{1}_{[h, \infty)}(t) h / t \leqslant 1$, the claim follows by the dominated convergence theorem.

Proof First note that by integration in polar coordinates one can check that the integral defining $g$ actually exists. Set $\epsilon>0$. Let $0<h<\operatorname{diam}(D)$ and $x$ and $z$ be arbitrary fixed points in $D$ such that $|x-z|=h$. Denote $j(x, y):=H(x, y)(f(y)-f(x))$. Observe that $|g(x)-g(z)|$ is bounded by the sum of two integrals $I_{1}$ and $I_{2}$ of $j(x, \cdot)-j(z, \cdot)$ over the sets $D \cap B(x, 2 h)$ and $D \backslash B(x, 2 h)$ respectively. On $D \cap B(x, 2 h)$ we have

$$
\begin{aligned}
I_{1} & =\left|\int_{D \cap B(x, 2 h)} H(x, y)(f(y)-f(x)) \mathrm{d} y-\int_{D \cap B(x, 2 h)} H(z, y)(f(y)-f(z)) \mathrm{d} y\right| \\
& \leqslant \int_{D \cap B(x, 3 h)}|H(x, y)||f(y)-f(x)| \mathrm{d} y+\int_{D \cap B(z, 3 h)}|H(z, y)||f(y)-f(z)| \mathrm{d} y \\
& \leqslant 2 \int_{0}^{3 h} F(t) \omega_{f}(t, D) t^{d-1} \mathrm{~d} t<\frac{\epsilon}{3}
\end{aligned}
$$

for sufficiently small $h$. Obviously $I_{2} \leqslant I_{3}+I_{4}$, where

$$
\begin{aligned}
& I_{3}:=\left|\int_{D \backslash B(x, 2 h)}(f(y)-f(z))(H(x, y)-H(z, y)) \mathrm{d} y\right|, \\
& I_{4}:=|f(z)-f(x)|\left|\int_{D \backslash B(x, 2 h)} H(x, y) \mathrm{d} y\right| .
\end{aligned}
$$

By the mean value theorem,

$$
I_{3} \leqslant|x-z| \sum_{i=1}^{d} \int_{D \backslash B(x, 2 h)}\left|H_{x_{i}}(\tilde{x}, y)\right||f(y)-f(z)| \mathrm{d} y
$$

for some $\tilde{x}=\theta x+(1-\theta) z, \theta \in(0,1)$. Note that for $y \in D \backslash B(x, 2 h)$ we have $|x-y| \geqslant$ $2|x-z|=2 h>0$. It follows that $|\tilde{x}-y| \geqslant h$ and consequently $|z-y| \leqslant|z-\tilde{x}|+|\tilde{x}-y| \leqslant$ $2|\tilde{x}-y|$. Thus,

$$
\begin{aligned}
I_{3} & \leqslant c_{d} h \int_{D \backslash B(x, 2 h)} \frac{F(|\tilde{x}-y|)}{|\widetilde{x}-y|}|f(y)-f(z)| \mathrm{d} y \\
& \leqslant c_{d} h \int_{D \backslash B(x, 2 h)} \frac{F(|z-y| / 2)}{|z-y|}|f(y)-f(z)| \mathrm{d} y \\
& \leqslant c_{d} h \int_{D \backslash B(z, h)} \frac{F(|z-y| / 2)}{|z-y|}|f(y)-f(z)| \mathrm{d} y \\
& \leqslant c_{d} h \int_{h}^{\operatorname{diam}(D)} F(t / 2) \omega_{f}(t, D) t^{d-2} \mathrm{~d} t \\
& \leqslant c_{d} h \int_{h / 2}^{\operatorname{diam}(D) / 2} F(t) \omega_{f}(2 t, D) t^{d-2} \mathrm{~d} t .
\end{aligned}
$$

Thus, by Remark 4.2 we see that $I_{3}<\epsilon / 3$ for sufficiently small $h$. Finally, Eq. 4.1 implies

$I_{4} \leqslant \omega_{f}(h, D) \int_{D \backslash B(x, 2 h)} F(|x-y|) \mathrm{d} y=\int_{0}^{\operatorname{diam}(D)} \mathbf{1}_{[2 h, \infty)}(t) F(t) \frac{\omega_{f}(h, D)}{\omega_{f}(t, D)} \omega_{f}(t, D) t^{d-1} \mathrm{~d} t$. 
Observe that $\mathbf{1}_{[2 h, \infty)}(t) \frac{\omega_{f}(h, D)}{\omega_{f}(t, D)} \leqslant 1$ by monotonicity of $\omega_{f}(\cdot, D)$. Thus, Eq. 4.1 justifies the application of the dominated convergence theorem and we obtain

$$
\lim _{h \rightarrow 0^{+}} \omega_{f}(h, D) \int_{D \backslash B(x, 2 h)} F(|x-y|) \mathrm{d} y=0 .
$$

In particular, $I_{4} \leqslant \epsilon / 3$ for sufficiently small $h$. It follows that $|g(x)-g(z)|<\epsilon$, if $h$ is sufficiently small. Thus, $g$ is uniformly continuous.

Lemma 4.3 Suppose $f$ is a uniformly continuous function on $D$ and $H(x, y)$ is a continuous function for $x, y \in D, x \neq y$, such that $\int_{D} H(x, y) \mathrm{d} y$ is continuously differentiable with respect to $x$. Assume there exists a non-increasing function $F:(0, \infty) \mapsto[0, \infty)$ such that for $i, j=1, \ldots, d$

$$
|H(x, y)|,\left|\frac{\partial H(x, y)}{\partial x_{i}}\right| \leqslant F(|x-y|), \quad\left|\frac{\partial^{2} H(x, y)}{\partial x_{i} \partial x_{j}}\right| \leqslant \frac{F(|x-y|)}{|x-y|} .
$$

If the following holds

$$
\int_{0}^{1 / 2} F(t) \omega_{f}(t, D) t^{d-1} d t<\infty
$$

then $u(x)=\int_{D} H(x, y) f(y) \mathrm{d} y$ is continuously differentiable with respect to $x \in D$ and

$$
\frac{\partial u(x)}{\partial x_{i}}=\int_{D} \frac{\partial H(x, y)}{\partial x_{i}}(f(y)-f(x)) \mathrm{d} y+f(x) \frac{\partial}{\partial x_{i}} \int_{D} H(x, y) \mathrm{d} y, \quad x \in D, \quad i=1, \ldots, d .
$$

Proof We will show that Eq. 4.4 holds for $x \in B(\bar{x}, r)$, where $r>0$ is such that $B(\bar{x}, 4 r) \subset$ $D$. By [34] we may and do identify $f$ with its uniformly continuous extension to $\mathbb{R}^{d}$ and for $\epsilon<r$ we set $f_{\epsilon}=\phi_{\epsilon} * f$, where $\phi_{\epsilon}$ are standard mollifiers. Note that for any $t>0$,

$$
\omega_{f_{\epsilon}}(t, B(\bar{x}, 2 r)) \leqslant \omega_{f}(t, D) .
$$

For $x \in D$ we define $u_{\epsilon}(x)=\int_{D} H(x, y) f_{\epsilon}(y) \mathrm{d} y$. From boundedness of $f_{\epsilon}$ we see that the integral defining $u_{\epsilon}$ is well defined and by the dominated convergence theorem $u_{\epsilon}(x) \rightarrow$ $u(x)$ for $x \in D$, as $\epsilon \rightarrow 0^{+}$. By Lemma 4.1 applied to $\frac{\partial H(x, y)}{\partial x_{i}}$ we have that the function

$$
\int_{D} \frac{\partial H(x, y)}{\partial x_{i}}\left(f_{\epsilon}(y)-f_{\epsilon}(x)\right) \mathrm{d} y+f_{\epsilon}(x) \frac{\partial}{\partial x_{i}} \int_{D} H(x, y) \mathrm{d} y
$$

is continuous on $D$. Let $x \in B(\bar{x}, r)$. By integrating (4.6) with respect to $x_{i}$ from $\bar{x}_{i}$ to $x_{i}$ we obtain a continuously differentiable function $\Psi_{\epsilon}(x)$ with respect to $x_{i}$ with Eq. 4.6 being its derivative. Denote $x=\left(\tilde{x}, x_{d}\right)$ and $\bar{x}=\left(\tilde{x}, \bar{x}_{d}\right)$, where $\tilde{x}=\left(x_{1}, \ldots, x_{d-1}\right)$ and $\bar{x}_{d}$ is fixed. The Fubini theorem and integration by parts yield

$$
\begin{aligned}
\Psi_{\epsilon}(x)= & \int_{\bar{x}_{d}}^{x_{d}}\left(\int_{D} \frac{\partial H(\tilde{x}, s, y)}{\partial s}\left(f_{\epsilon}(y)-f_{\epsilon}(\tilde{x}, s)\right) \mathrm{d} y+f_{\epsilon}(\tilde{x}, s) \frac{\partial}{\partial s} \int_{D} H(\tilde{x}, s, y) \mathrm{d} y\right) \mathrm{d} s \\
= & \left.\int_{D} H(\tilde{x}, s, y)\left(f_{\epsilon}(y)-f_{\epsilon}(\tilde{x}, s)\right)\right|_{\bar{x}_{d}} ^{x_{d}} \mathrm{~d} y-\int_{D} \int_{\bar{x}_{d}}^{x_{d}} H(\tilde{x}, s, y) \frac{\partial}{\partial s}\left(f_{\epsilon}(y)-f_{\epsilon}(\tilde{x}, s)\right) \mathrm{d} s \mathrm{~d} y \\
& +\left.f_{\epsilon}(\tilde{x}, s) \int_{D} H(\tilde{x}, s, y) \mathrm{d} y\right|_{\bar{x}_{d}} ^{x_{d}}-\int_{\bar{x}_{d}}^{x_{d}} \frac{\partial f_{\epsilon}(\tilde{x}, s)}{\partial s} \int_{D} H(\tilde{x}, s, y) \mathrm{d} y \mathrm{~d} s=u_{\epsilon}(x)-u_{\epsilon}(\bar{x}) .
\end{aligned}
$$

Thus, for $x \in B(\bar{x}, r)$ the partial derivative $\frac{\partial u_{\epsilon}(x)}{\partial x_{d}}$ exists and is equal to Eq. 4.6. The same argument applies to any $i=1, \ldots, d$. It remains to prove that Eq. 4.6 converges uniformly to 
Eq. 4.4, as $\epsilon \rightarrow 0^{+}$. Since $f_{\epsilon} \rightarrow f$ uniformly as $\epsilon \rightarrow 0^{+}$, it is enough to prove the convergence of first integral in Eq. 4.6. Fix $\delta>0$. Since $\int_{0}^{\operatorname{diam}(D)} F(t) \omega_{f}(t, D) t^{d-1} \mathrm{~d} t<\infty$, there is $\gamma>0$ such that $\int_{0}^{\gamma} F(t) \omega_{f}(t, D) t^{d-1} \mathrm{~d} t<\delta /\left(4\left|\mathbb{S}^{n-1}\right|\right)$. Invoking Eq. 4.5 we get

$$
\begin{aligned}
& \left|\int_{B(x, \gamma)} \frac{\partial H(x, y)}{\partial x_{i}}\left(f_{\epsilon}(y)-f_{\epsilon}(x)\right) \mathrm{d} y-\int_{B(x, \gamma)} \frac{\partial H(x, y)}{\partial x_{i}}(f(y)-f(x)) \mathrm{d} y\right| \\
\leqslant & 2\left|\int_{B(x, \gamma)} \frac{\partial H(x, y)}{\partial x_{i}} \omega_{f}(|x-y|, D) \mathrm{d} y\right| \leqslant 2\left|\mathbb{S}^{n-1}\right| \int_{0}^{\gamma} F(t) \omega_{f}(t, D) t^{d-1} \mathrm{~d} t<\frac{\delta}{2} .
\end{aligned}
$$

On the complement of $B(x, \gamma)$, the function $\left|\frac{\partial H(x, y)}{\partial x_{i}}\right|$ is bounded by some constant $C>0$. Choose $\epsilon_{0}>0$ such that $\left\|f_{\epsilon}-f\right\|_{\infty} \leqslant \delta /(4 C|D|)$ for $\epsilon<\epsilon_{0}$. Then

$$
\left|\int_{D \backslash B(x, \gamma)} \frac{\partial H(x, y)}{\partial x_{i}}\left(f_{\epsilon}(y)-f_{\epsilon}(x)-f(y)+f(x)\right) \mathrm{d} y\right| \leqslant 2 \frac{\delta}{4 C|D|}|D| C=\frac{\delta}{2},
$$

which combined with Eq. 4.7 and arbitrary choice of $\delta$ ends the proof.

Now we are ready to prove Theorem 1.2.

Proof of Theorem 1.2 Let $u \in \mathcal{L}^{1}$ be a distributional solution of Eq. 1.7. By Theorem 1.1, $u+G_{D}[f]$ satisfies the mean-value property inside $D$. Moreover, by the proof of Lemma 3.5 we get that $G_{D}[f] \in L^{1}(D)$ and in view of $f$ vanishing on $D^{c}$ we in fact obtain that $G_{D}[f] \in \mathcal{L}^{1}$. Thus, by Lemma $2.2, u+G_{D}[f] \in C^{2}(D)$, and it remains to prove that $G_{D}[f] \in C^{2}(D)$. By the Hunt formula Eq. 2.4,

$$
G_{D}[f](x)=\int_{D} G(x, y) f(y) \mathrm{d} y-\int_{D} \mathbb{E}^{x} G\left(X_{\tau_{D}}, y\right) f(y) \mathrm{d} y=: I_{1}(x)+I_{2}(x) .
$$

First, we verify that the separation is justified. Observe that $G_{D}[f]$ is finite for a.e. $x \in D$. Moreover, since both $G$ and $G_{D}$ are symmetric, we get that $(x, y) \mapsto \mathbb{E}^{x} G\left(X_{\tau_{D}}, y\right)$ is also symmetric. Thus,

$$
\mathbb{E}^{x} G\left(X_{\tau_{D}}, y\right)=\mathbb{E}^{y} G\left(X_{\tau_{D}}, x\right) .
$$

Using $(\mathrm{G})$ we obtain that both $G$ and its first and second derivative are bounded either by $S\left(\delta_{D}(x)\right)$ or $S\left(\delta_{D}(x)\right) / \delta_{D}(x)$, depending on the finiteness of $\int_{0}^{1 / 2}\left|G^{\prime}(t)\right| t^{d-1} \mathrm{~d} t$, and consequently, $I_{2}(x)$ is also finite. It follows that $I_{1}(x)$ must be finite as well. Furthermore, by the same estimate we are allowed to differentiate under the integral sign. Hence, $x \mapsto$ $\mathbb{E}^{x} G\left(X_{\tau_{D}}, y\right)$ belongs to $C^{2}(D)$ and consequently, $I_{2} \in C^{2}(D)$. Thus, it is enough to prove that $I_{1}$ is in $C^{2}(D)$. Fix $i, j \in\{1, \ldots, d\}$. Consider two cases.

1. Let $\int_{0}^{1}\left|G^{\prime}(t)\right| t^{d-1} \mathrm{~d} t=\infty$. Fix $x \in D$. From Lemma 2.6 we get

$$
\begin{aligned}
\frac{\partial}{\partial x_{i}} I_{1}(x) & =\int_{\mathbb{R}^{d}} G(x-y) \frac{\partial}{\partial x_{i}}\left(f \chi_{1}\right)(y) \mathrm{d} y+\int_{\mathbb{R}^{d}} \frac{\partial}{\partial x_{i}}\left(G \chi_{2}\right)(x-y)\left(f \mathbf{1}_{D}\right)(y)\left(1-\chi_{1}\right)(y) \mathrm{d} y \\
& =: w_{1}(x)+w_{2}(x),
\end{aligned}
$$

where the localization functions $\chi_{1}$ and $\chi_{2}$ are chosen in dependence of $x$. Note that in the integral defining $w_{2}$, due to the function $\chi_{2}$ and $(\mathrm{G})$, integration w.r.t. $y$ takes place in a region where $G$ and its derivative are bounded. Hence, from $(G)$ we see that differentiating under the integral sign is justified. We obtain

$$
\frac{\partial}{\partial x_{j}} w_{2}(x)=\int_{\mathbb{R}^{d}} \frac{\partial^{2}}{\partial x_{i} \partial x_{j}}\left(G \chi_{2}\right)(x-y)\left(f \mathbf{1}_{D}\right)(y)\left(1-\chi_{1}\right)(y) \mathrm{d} y .
$$


If we split $w_{1}$ into two integrals

$$
\begin{aligned}
w_{1}(x) & =\int_{D_{1}} G(x-y) \frac{\partial}{\partial y_{i}}\left(f \chi_{1}\right)(y) \mathrm{d} y+\int_{D \backslash D_{1}} G(x-y) \frac{\partial}{\partial y_{i}}\left(f \chi_{1}\right)(y) \mathrm{d} y \\
& =: w_{3}(x)+w_{4}(x),
\end{aligned}
$$

where $x \in D_{1} \subset D$ is such that $\left.\chi_{1}\right|_{D_{1}} \equiv 1$ then the same argument can be applied to $w_{4}$. Thus,

$$
\frac{\partial}{\partial x_{j}} w_{4}(x)=\int_{D \backslash D_{1}} \frac{\partial}{\partial x_{j}} G(x-y) \frac{\partial}{\partial y_{i}}\left(f \chi_{1}\right)(y) \mathrm{d} y .
$$

Next, observe that

$$
\int_{0}^{\operatorname{diam}\left(D_{1}\right)} S(t) \omega_{\nabla f}\left(t, D_{1}\right) t^{d-1} \mathrm{~d} t \leqslant \int_{0}^{\operatorname{diam}(D)} S(t) \omega_{\nabla f}(t, D) t^{d-1} \mathrm{~d} t<\infty .
$$

Moreover, by Corollary 2.7 the function $x \mapsto \int_{D_{1}} G(x, y) d y$ is continuously differentiable and from (G) we see that Eq. 4.2 of Lemma 4.3 is satisfied for $H(x, y)=$ $G(|x-y|)$ and $F=S$. Hence, in view of Proposition 2.5, for $h(x)=\frac{\partial}{\partial x_{i}} f(x)$ we obtain

$$
\frac{\partial}{\partial x_{j}} w_{3}(x)=\int_{D_{1}} \frac{\partial G(x, y)}{\partial x_{j}}(h(y)-h(x)) \mathrm{d} y+h(x) \frac{\partial}{\partial x_{i}} \int_{D_{1}} G(x, y) \mathrm{d} y .
$$

2. Now let $\int_{0}^{1}\left|G^{\prime}(t)\right| t^{d-1} \mathrm{~d} t<\infty$. In this case, by the Fubini theorem and the fundamental theorem of calculus we get

$$
\frac{\partial}{\partial x_{i}} \int_{D} G(x, y) f(y) \mathrm{d} y=\int_{D} \frac{\partial G(x, y)}{\partial x_{i}} f(y) \mathrm{d} y .
$$

A similar argument applied to $H(x, y)=\frac{\partial G(x, y)}{\partial x_{i}}$ shows that the assumptions of Lemma 4.3 are satisfied with $F=S$. Note that here we use the additional assumption on $G^{\prime \prime \prime}$. Thus,

$$
\frac{\partial^{2}}{\partial x_{i} \partial x_{j}} \int_{D} G(x, y) f(y) \mathrm{d} y=\int_{D} \frac{\partial^{2} G(x, y)}{\partial x_{i} \partial x_{j}}(f(y)-f(x)) \mathrm{d} y+f(x) \frac{\partial}{\partial x_{j}} \int_{D} \frac{\partial G(x, y)}{\partial x_{i}} \mathrm{~d} y,
$$

and the proof is completed.

\section{Counterexamples for the Case " $\alpha+\beta=2$ "}

In this section we provide several counterexamples for Theorem 1.2. These examples are of the nature, $\alpha+\beta=2$ ", i.e., for $\alpha \in(0,2)$ we give a function $f \in C^{2-\alpha}(D)$ for which the solution of the Dirichlet problem Eq. 5.1 is not twice continuously differentiable inside of $D$. In Section 6 we explain how the counterexamples can be modified in order to match the assumptions of Theorem 1.2.

Let $D=B_{1}$. Consider a Dirichlet problem

$$
\begin{aligned}
\Delta^{\alpha / 2} u & =f \text { in } B_{1}, \\
u & =0 \text { in } B_{1}^{c},
\end{aligned}
$$


where $\alpha \in(0,2)$. It is known (see [7] or Theorem 1.1) that $u(x)=\int_{D} G_{B_{1}}(x, y) f(y) \mathrm{d} y$, where $G_{B_{1}}(x, y)$ is Green function for the operator $\Delta^{\alpha / 2}$ and domain $B_{1}$, solves Eq. 5.1. By the Hunt formula (2.4),

$$
G_{B_{1}}(x, y)=G(x, y)-\mathbb{E}^{x} G\left(X_{\tau_{B_{1}}}, y\right), \quad x, y \in B_{1},
$$

where $G$ is the (compensated) potential kernel for process $X_{t}$ whose generator is $\Delta^{\alpha / 2}$. We claim that for any fixed $y \in B_{1}$, the function $x \mapsto \mathbb{E}^{x} G\left(X_{\tau_{D}}, y\right)$ belongs to $C^{\infty}\left(B_{1}\right)$. Indeed, using the explicit formula for the Poisson kernel for the unit ball [5], we get

$$
\mathbb{E}^{x} G\left(X_{\tau_{B_{1}}}, y\right)=c_{d, \alpha} \int_{B_{1}^{c}} G(z, y)\left(\frac{1-|x|^{2}}{|z|^{2}-1}\right)^{\alpha / 2} \frac{\mathrm{d} z}{|x-z|^{d}} .
$$

Observe that $|z-y|>\delta_{B_{1}}(y)$, which implies that we are separated from the origin. It follows that $B_{1}^{c} \ni z \mapsto G(z, y)$ is locally bounded and since the integral converges at infinity for any form of $G$, we obtain that for fixed $y \in B_{1}$, the function $B_{1} \ni x \mapsto \mathbb{E}^{x} G\left(X_{\tau_{B_{1}}}, y\right)$ is bounded. Since it has the mean-value property in $B_{1}$, by [20, Theorem 1.7 and Remark $1.8(\mathrm{~b})]$ it is smooth. Thus, the regularity problem is reduced to the regularity of the function $x \mapsto w(x)=\int_{B_{1}} G(x, y) f(y) \mathrm{d} y=G * f(x)$ (we adopt a convention that $f \equiv 0$ outside $\left.B_{1}\right)$.

\subsection{Case $\alpha \in(0,1)$}

We follow closely the idea from the proof of Theorem 1.2 apart from the fact that at the end we will show that the last function $w_{3}$ is not continuously differentiable. From Lemma 2.6 we get

$$
\begin{aligned}
\frac{\partial}{\partial x_{d}} w(x) & =\int_{\mathbb{R}^{d}} G(x-y) \frac{\partial}{\partial y_{d}}\left(f \chi_{1}\right)(y) \mathrm{d} y+\int_{\mathbb{R}^{d}} \frac{\partial}{\partial x_{d}}\left(G \chi_{2}\right)(x-y)\left(f \mathbf{1}_{B_{1}}\right)(y)\left(1-\chi_{1}\right)(y) \mathrm{d} y \\
& =: w_{1}(x)+w_{2}(x),
\end{aligned}
$$

if only $f \in C_{b}^{1}\left(B_{1}\right) . \chi_{1}$ and $\chi_{2}$ in Eq. 5.2 are chosen for $x_{0}=0$. Put $f(y)=\left(\left(y_{d}\right)_{+}\right)^{2-\alpha}$ and calculate $\frac{\partial^{2}}{\partial x_{d}^{2}} w(x)$ in $x=0$. Since in $w_{2}$ we are separated from the origin, it follows that

$$
\frac{\partial}{\partial x_{d}} w_{2}(x)=\int_{\mathbb{R}^{d}} \frac{\partial^{2}}{\partial x_{d}^{2}}\left(G \chi_{2}\right)(x-y)\left(f \mathbf{1}_{B_{1}}\right)(y)\left(1-\chi_{1}\right)(y) \mathrm{d} y .
$$

If we split $w_{1}$ into

$$
\begin{aligned}
w_{1}(x)= & \int_{B_{1 / 4}} G(x-y) \frac{\partial}{\partial y_{d}}\left(f \chi_{1}\right)(y) \mathrm{d} y \\
& +\int_{B_{1 / 4}^{c}} G(x-y) \frac{\partial}{\partial y_{d}}\left(f \chi_{1}\right)(y) \mathrm{d} y=: w_{3}(x)+w_{4}(x),
\end{aligned}
$$

then the same argument applies for $w_{4}$. Therefore, it remains to calculate the derivative of $w_{3}$. Observe that on $B_{1 / 4}$ we have $f \chi_{1} \equiv f$. To simplify the notation we accept a mild 
ambiguity and by $h$ we denote, depending on the context, either a real number or a vector in $\mathbb{R}^{d}$ of the form $(0, \ldots, 0, h)$. Let $h>0$.

$$
\begin{aligned}
\frac{1}{-h}\left(w_{3}(-h)-w_{3}(0)\right) & =\frac{2-\alpha}{-h} \int_{B_{1 / 4}}\left(|-h-y|^{\alpha-d}-|y|^{\alpha-d}\right)\left(\left(y_{d}\right)_{+}\right)^{1-\alpha} \mathrm{d} y= \\
& =(2-\alpha) \int_{A} \frac{|y|^{\alpha-d}-|y+h|^{\alpha-d}}{h} y_{d}^{1-\alpha} \mathrm{d} y=:(2-\alpha) I(h),
\end{aligned}
$$

where $A=B_{1 / 4} \cap\left\{y_{d}>c|y|\right\}$ for some $c \in(0,1)$. By the Fatou lemma,

$$
\begin{aligned}
\liminf _{h \rightarrow 0^{+}} I(h) & \geqslant \int_{A} \liminf _{h \rightarrow 0^{+}} \frac{|y|^{-d+\alpha}-|y+h|^{-d+\alpha}}{h} y_{d}^{1-\alpha} \mathrm{d} y=c_{d, \alpha} \int_{A} \frac{y_{d}}{|y|^{d+2-\alpha}} y_{d}^{1-\alpha} \mathrm{d} y \\
& \geqslant c_{d, \alpha} \int_{A} \frac{|y|}{|y|^{d+2-\alpha}}|y|^{1-\alpha} \mathrm{d} y=c_{d, \alpha} \int_{0}^{1 / 4} \frac{\mathrm{d} t}{t} .
\end{aligned}
$$

Hence $\frac{\partial^{2}}{\partial x_{d}{ }^{2}} w_{-}(0)=\infty$.

\subsection{Case $\alpha=1$}

Let $d=1$. The compensated kernel is of the form $G(x, y)=\frac{1}{\pi} \ln \frac{1}{|x-y|}$. Note that we cannot apply [12, Lemma 2.3] because (ii) does not hold. Instead let us write

$$
\begin{aligned}
\frac{w(x+h)-w(x)}{h}= & \int_{-1}^{1} \frac{G(x+h-y)-G(x-y)}{h}(f(y)-f(x)) \mathrm{d} y \\
& +f(x) \int_{-1}^{1} \frac{G(x+h-y)-G(x-y)}{h} \mathrm{~d} y .
\end{aligned}
$$

Let $f$ be a Lipschitz function. We claim that

$$
\lim _{h \rightarrow 0} \int_{-1}^{1} \frac{G(x+h-y)-G(x-y)}{h}(f(y)-f(x)) \mathrm{d} y=\int_{-1}^{1} G^{\prime}(x-y)(f(y)-f(x)) \mathrm{d} y .
$$

Indeed, we have

$$
G(x+h-y)-G(x-y)=-\frac{1}{\pi} \ln \left|1+\frac{h}{x-y}\right| .
$$

Let us observe that the function $s(u):=u \ln \left|1+u^{-1}\right|, u \neq-1$, is integrable on the set $\left(-2,-\frac{2}{3}\right)$ and bounded on its complement. Denote $D_{h}=\left\{y \in(-1,1): \frac{h}{x-y} \in\right.$ $\left.\left(-\frac{3}{2},-\frac{1}{2}\right)\right\}$. Then, since $f$ is a Lipschitz function, we have

$$
\left|\frac{G(x+h-y)-G(x-y)}{h}(f(y)-f(x))\right| \leqslant c\left|s\left(\frac{x-y}{h}\right)\right|,
$$

for some constant $c>0$, and by change of variables we obtain

$$
\left|\int_{D_{h}} \frac{G(x+h-y)-G(x-y)}{h}(f(y)-f(x)) \mathrm{d} y\right| \leqslant c h \int_{-2}^{-2 / 3}|s(x)| \mathrm{d} x .
$$

Moreover, Eq. 5.4 justifies the application of the dominated convergence theorem to the function

$$
(-1,1) \ni y \mapsto \frac{G(x+h-y)-G(x-y)}{h}(f(y)-f(x)) \mathbf{1}_{D_{h}^{c}}(y),
$$


and Eq. 5.3 follows by Eq. 5.5.

Next, denote

$$
\begin{aligned}
F(x) & :=\int_{-1}^{1} G(x-y) \mathrm{d} y=-\frac{1}{\pi} \int_{-1}^{1} \ln |y-x| \mathrm{d} y=-\frac{1}{\pi} \int_{-1-x}^{1-x} \ln |s| \mathrm{d} s \\
& =-\frac{1}{\pi} \int_{0}^{1+x} \ln s \mathrm{~d} s-\frac{1}{\pi} \int_{0}^{1-x} \ln s \mathrm{~d} s .
\end{aligned}
$$

It follows that

$$
\lim _{h \rightarrow 0} \int_{-1}^{1} \frac{G(x+h-y)-G(x-y)}{h} \mathrm{~d} y=F^{\prime}(x)=\frac{1}{\pi} \ln \frac{1-x}{1+x} .
$$

Hence,

$$
w^{\prime}(x)=\int_{-1}^{1} G^{\prime}(x-y)(f(y)-f(x)) \mathrm{d} y+f(x) F^{\prime}(x) .
$$

Put $f(y)=y_{+} \ln ^{-\beta}\left(1+\left(y^{-1}\right)_{+}\right), \beta \in(0,1)$. Since $f \in C^{1}((-1,1))$, it is a Lipschitz function. Let $h<0$. Since $f(y)=0$ for $y \leqslant 0$, from Eq. 5.6 we obtain

$$
\begin{aligned}
\frac{1}{h}\left(w^{\prime}(h)-w^{\prime}(0)\right) & =\frac{1}{h} \int_{0}^{1}\left(\frac{1}{|h-y|}-\frac{1}{|y|}\right) y \ln ^{-\beta}\left(1+\frac{1}{y}\right) \mathrm{d} y \\
& =\frac{1}{h} \int_{0}^{1}\left(\frac{1}{y-h}-\frac{1}{y}\right) y \ln ^{-\beta}\left(1+\frac{1}{y}\right) \mathrm{d} y \\
& =\frac{1}{h} \int_{0}^{1} \frac{h}{y(y-h)} y \ln ^{-\beta}\left(1+\frac{1}{y}\right) \mathrm{d} y=\int_{0}^{1} \frac{1}{y-h} \ln ^{-\beta}\left(1+\frac{1}{y}\right) \mathrm{d} y .
\end{aligned}
$$

By the monotone convergence theorem,

$$
\int_{0}^{1} \frac{1}{y-h} \ln ^{-\beta}\left(1+\frac{1}{y}\right) \mathrm{d} y \stackrel{h \rightarrow 0^{-}}{\longrightarrow} \int_{0}^{1} \frac{1}{y} \ln ^{-\beta}\left(1+\frac{1}{y}\right) \mathrm{d} y .
$$

Since

$$
\lim _{y \rightarrow 0^{+}} \frac{\ln \left(1+\frac{1}{y}\right)}{\ln \frac{1}{y}}=1,
$$

we obtain $w_{-}^{\prime \prime}(0)=\infty$. For $d>1$ and $\beta \in(0,1)$ we apply [12, Lemma 2.3] to the function $f(y)=\left(y_{d}\right)_{+} \ln ^{-\beta}\left(1+\left(y_{d}^{-1}\right)_{+}\right)$and $G(x, y)=|x-y|^{-d+1}$, in order to obtain

$$
\frac{\partial}{\partial x_{d}} w(x)=\int_{B_{1}} \frac{\partial G(x, y)}{\partial x_{d}}(f(y)-f(x)) \mathrm{d} y+f(x) \frac{\partial}{\partial x_{d}} \int_{B_{1}} G(x, y) \mathrm{d} y .
$$

By Corollary 2.7, the condition (iii) of [12, Lemma 2.3] holds. Denote

$$
H(x, y):=\frac{\partial G(x, y)}{\partial x_{d}}=(1-d) \frac{(x-y)_{d}}{|x-y|^{d+1}}=-c_{d} \frac{(x-y)_{d}}{|x-y|^{d+1}},
$$

$c_{d}>0$. Let $h>0$ and $H_{0}(y)=H(0, y)$ for $y \in \mathbb{R}^{d}$. Note that $H_{0} \geqslant 0$ on the set $\left\{y \in B_{1}: y_{d} \geqslant 0\right\}$. We calculate the left-sided second partial derivative $\frac{\partial^{2}}{\partial x_{d}{ }^{2}} w(x)$ in $x=0$. 
Observe that $f(x)$ is equal to 0 for $x=0$ and $x=-h$, thus, the expression in the first term of Eq. 5.8 simplifies. Furthermore, the second term vanishes, and the remaining limit is

$$
\lim _{h \rightarrow 0^{+}}-\frac{1}{h} \int_{B_{1}}\left(H_{0}(y+h)-H_{0}(y)\right) f(y) \mathrm{d} y .
$$

Let $f_{1}(s)=f((0, \ldots, 0, s))$ and note that $f_{1}^{\prime} \geqslant 0$. We have

$$
\begin{aligned}
& \int_{B_{1}}\left(H_{0}(y+h)-H_{0}(y)\right) f(y) \mathrm{d} y=\int_{B_{1}}\left(H_{0}(y+h)-H_{0}(y)\right) f_{1}\left(y_{d}\right) \mathrm{d} y \\
= & \int_{B_{1}}\left(H_{0}(y+h)-H_{0}(y)\right) \int_{0}^{y_{d}} f_{1}^{\prime}(s) \mathrm{d} s \mathrm{~d} y \\
= & \int_{0}^{1} \mathrm{~d} s f_{1}^{\prime}(s) \int_{B_{1} \cap \mathbb{H}_{s}}\left(H_{0}(y+h)-H_{0}(y)\right) \mathrm{d} y,
\end{aligned}
$$

where $\mathbb{H}_{s}=\left\{y: y_{d}>s\right\}$. Denote $\tilde{y}=\left(y_{1}, \ldots, y_{d-1}\right) \in \mathbb{R}^{d-1}$. Then

$$
\begin{aligned}
& \int_{0}^{1} \mathrm{~d} s f_{1}^{\prime}(s) \int_{B_{1} \cap \mathbb{H}_{s}}\left(H_{0}(y+h)-H_{0}(y)\right) \mathrm{d} y \\
= & \int_{0}^{1} \mathrm{~d} s f_{1}^{\prime}(s) \int_{|\tilde{y}|<1} \mathrm{~d} \tilde{y} \int_{s}^{\sqrt{1-|\tilde{y}|^{2}}}\left(H_{0}(y+h)-H_{0}(y)\right) \mathrm{d} y_{d} \\
= & \int_{0}^{1} \mathrm{~d} s f_{1}^{\prime}(s) \int_{|\tilde{y}|<1} \mathrm{~d} \tilde{y}\left(\int_{s+h}^{\sqrt{1-|\tilde{y}|^{2}}+h} H_{0}(y) \mathrm{d} y_{d}-\int_{s}^{\sqrt{1-|\tilde{y}|^{2}}} H_{0}(y) \mathrm{d} y_{d}\right) \\
= & \int_{0}^{1} \mathrm{~d} s f_{1}^{\prime}(s) \int_{|\tilde{y}|<1} \mathrm{~d} \tilde{y}\left(\int_{\sqrt{1-|\tilde{y}|^{2}}}^{\sqrt{1-|\tilde{y}|^{2}}+h} H_{0}(y) \mathrm{d} y_{d}-\int_{s}^{s+h} H_{0}(y) \mathrm{d} y_{d}\right) \\
= & \int_{0}^{1} \mathrm{~d} s f_{1}^{\prime}(s) \int_{|\tilde{y}|<1}\left(G\left(\left(\tilde{y}, \sqrt{1-|\tilde{y}|^{2}}\right)\right)-G\left(\left(\tilde{y}, \sqrt{1-|\tilde{y}|^{2}}+h\right)\right)\right) \mathrm{d} \tilde{y} \\
& -\int_{0}^{1} \mathrm{~d} s f_{1}^{\prime}(s) \int_{|\tilde{y}|<1}(G((\tilde{y}, s))-G((\tilde{y}, s+h))) \mathrm{d} \tilde{y} \\
= & : I_{1}(h)-I_{2}(h) .
\end{aligned}
$$

Let $h \in\left(0, \frac{1}{2}\right)$. By the mean value theorem, for every $|\tilde{y}|<1$ there is $\theta_{\tilde{y}} \in(0,1)$ such that

$$
\left|\frac{G\left(\left(\tilde{y}, \sqrt{1-|\tilde{y}|^{2}}+h\right)\right)-G\left(\left(\tilde{y}, \sqrt{1-|\tilde{y}|^{2}}\right)\right)}{h}\right| \leqslant H_{0}\left(\left(\tilde{y}, \sqrt{1-|\tilde{y}|^{2}}+\theta_{\tilde{y}} h\right)\right) \leqslant 2 c_{d} .
$$

Moreover, if we denote $E=\left\{y \in \mathbb{R}^{d}:|\tilde{y}|<1\right.$ and $\left.y_{d} \in(0,1)\right\}$, then we have

$$
\int_{E} f_{1}^{\prime}\left(y_{d}\right) \mathrm{d} y<\infty .
$$


Thus, by the dominated convergence theorem,

$$
\begin{aligned}
\lim _{h \rightarrow 0^{+}} & \int_{0}^{1} \mathrm{~d} s f_{1}^{\prime}(s) \int_{|\tilde{y}|<1} \frac{G\left(\left(\tilde{y}, \sqrt{1-|\tilde{y}|^{2}}+h\right)\right)-G\left(\left(\tilde{y}, \sqrt{1-|\tilde{y}|^{2}}\right)\right)}{h} \mathrm{~d} \tilde{y} \\
\quad= & \int_{0}^{1} \mathrm{~d} s f_{1}^{\prime}(s) \int_{|\tilde{y}|<1} \lim _{h \rightarrow 0^{+}} \frac{G\left(\left(\tilde{y}, \sqrt{1-|\tilde{y}|^{2}}+h\right)\right)-G\left(\left(\tilde{y}, \sqrt{1-|\tilde{y}|^{2}}\right)\right)}{h} \mathrm{~d} \tilde{y} \\
= & \int_{0}^{1} \mathrm{~d} s f_{1}^{\prime}(s) \int_{|\tilde{y}|<1} H_{0}\left(\left(\tilde{y}, \sqrt{1-|\tilde{y}|^{2}}\right)\right) \mathrm{d} \tilde{y} \\
= & \int_{E} H_{0}\left(\left(\tilde{y}, \sqrt{1-|\tilde{y}|^{2}}\right)\right) f_{1}^{\prime}\left(y_{d}\right) \mathrm{d} y
\end{aligned}
$$

It follows that

$$
\lim _{h \rightarrow 0^{+}} \frac{\left|I_{1}(h)\right|}{h}<\infty .
$$

Next, we note that since $G$ is radially non-increasing, $h>0$ and $f_{1}^{\prime} \geqslant 0$, the integrand of $I_{2}$ has a constant, positive sign. Hence, by the Fatou lemma,

$$
\begin{aligned}
\liminf _{h \rightarrow 0^{+}} & \int_{0}^{1} \mathrm{~d} s f_{1}^{\prime}(s) \int_{|\tilde{y}|<1} \frac{G((\tilde{y}, s))-G((\tilde{y}, s+h))}{h} \mathrm{~d} \tilde{y} \\
& \geqslant \int_{0}^{1} \mathrm{~d} s f_{1}^{\prime}(s) \int_{|\tilde{y}|<1} H_{0}((\tilde{y}, s)) \mathrm{d} \tilde{y}=\int_{E} H_{0}(y) f_{1}^{\prime}\left(y_{d}\right) \mathrm{d} y .
\end{aligned}
$$

We have

$$
f_{1}^{\prime}(s)=\ln ^{-\beta}\left(1+s^{-1}\right)+\frac{\beta}{s+1} \ln ^{-\beta-1}\left(1+s^{-1}\right), \quad s>0 .
$$

Thus,

$$
\begin{aligned}
& \int_{E} \frac{y_{d}}{|y|^{d+1}} \ln ^{-\beta}\left(1+\left(y_{d}^{-1}\right)_{+}\right) \mathrm{d} y \geqslant \int_{A} \frac{y_{d}}{|y|^{d+1}} \ln ^{-\beta}\left(1+y_{d}^{-1}\right) \mathrm{d} y \\
& \geqslant c_{d} \int_{A} \frac{|y|}{|y|^{d+1}} \ln ^{-\beta}\left(1+(c|y|)^{-1}\right) \mathrm{d} y \geqslant c_{d} \int_{0}^{1 / 4} \frac{1}{t} \ln ^{-\beta}\left(1+(c t)^{-1}\right) \mathrm{d} t .
\end{aligned}
$$

Hence, $\frac{\partial^{2}}{\partial x_{d}^{2}} w_{-}(0)=\infty$.

\subsection{Case $\alpha \in(1,2)$}

Let $d=1$. The compensated potential kernel is of the form $G(x, y)=c_{\alpha}|x-y|^{\alpha-1}$. By [12, Lemma 2.1], for every bounded $f$ we have

$$
w^{\prime}(x)=\int_{-1}^{1} G^{\prime}(x-y) f(y) \mathrm{d} y .
$$

We calculate the second derivative of $w(x)$ for $|x|<1$. Observe that

$$
I_{1}(x):=\frac{\mathrm{d}}{\mathrm{d} x} \int_{\substack{|y|<1,|y-x|>\frac{1}{2}}} G^{\prime}(y-x) f(y) \mathrm{d} y=\int_{\substack{|y|<1,|y-x|>\frac{1}{2}}} G^{\prime \prime}(y-x) f(y) \mathrm{d} y .
$$


Hence $w^{\prime \prime}(x)=I_{1}(x)+I_{2}(x)$, where

$$
I_{2}(x):=\lim _{h \rightarrow 0} \int_{|y-x|<\frac{1}{2}}=\frac{G^{\prime}(y-x-h)-G^{\prime}(y-x)}{h} f(y) \mathrm{d} y .
$$

Put $f(y)=\left(y_{+}\right)^{2-\alpha}$. Then

$$
I_{2}(0)=\lim _{h \rightarrow 0} \int_{0}^{1 / 2} \frac{G^{\prime}(y-h)-G^{\prime}(y)}{h} y^{2-\alpha} \mathrm{d} y .
$$

We compute the left-sided limit. Let $h>0$.

$$
\begin{aligned}
\int_{0}^{1 / 2} \frac{G^{\prime}(y+h)-G^{\prime}(y)}{-h} y^{2-\alpha} \mathrm{d} y & =c_{\alpha} \int_{0}^{1 / 2} \frac{y^{\alpha-2}-(y+h)^{\alpha-2}}{h} y^{2-\alpha} \mathrm{d} y \\
& =c_{\alpha} \int_{0}^{1 / 2} \frac{1-(1+h / y)^{\alpha-2}}{h} \mathrm{~d} y \\
& =c_{\alpha} \int_{0}^{1 /(2 h)}\left(1-\left(1+y^{-1}\right)^{\alpha-2}\right) \mathrm{d} y \\
& =c_{\alpha} \int_{2 h}^{\infty}\left(1-(1+s)^{\alpha-2}\right) \frac{\mathrm{d} s}{s^{2}} .
\end{aligned}
$$

Thus, $w_{-}^{\prime \prime}(0)=\infty$. Now let $d>1$. Then $G(x, y)=c_{d, \alpha}|x-y|^{-d+\alpha}$ for some $c_{d, \alpha}>0$. [12, Lemma 2.1] implies

$$
\frac{\partial w(x)}{\partial x_{d}}=\int_{B_{1}} \frac{\partial G(x, y)}{\partial x_{d}} f(y) \mathrm{d} y,
$$

where $f(y)=\left(\left(y_{d}\right)_{+}\right)^{2-\alpha}$. We follow closely the argumentation from the case $\alpha=1$, $d>1$. We introduce the same notation

$$
H(x, y):=\frac{\partial G(x, y)}{\partial x_{d}}=-c_{d, \alpha}(d-\alpha) \frac{(x-y)_{d}}{|x-y|^{d+2-\alpha}}=-\tilde{c}_{d, \alpha} \frac{(x-y)_{d}}{|x-y|^{d+\beta}},
$$

$\tilde{c}_{d, \alpha}>0, \beta:=2-\alpha \in(0,1)$. By repeating (5.9) - (5.12) we conclude that it remains to calculate

$$
\int_{E}-H_{0}(y) f_{1}^{\prime}\left(y_{d}\right) \mathrm{d} y
$$

where, as before, $f_{1}(h)=f((0, \ldots, 0, h))$. Here we have $f_{1}^{\prime}(h)=(2-\alpha) h^{1-\alpha}$. Note that the argumentation (5.9) - (5.12) is correct even though $f_{1}$ does not belong to $C^{1}\left(B_{1}\right)$ for $\alpha>1$. We obtain

$$
\int_{E} \frac{y_{d}}{|y|^{d+\beta}}\left(y_{d}\right)_{+}^{1-\alpha} \mathrm{d} y \geqslant \int_{A} \frac{y_{d}}{|y|^{d+\beta}} y_{d}^{1-\alpha} \mathrm{d} y \geqslant c_{\alpha} \int_{A} \frac{|y|}{|y|^{d+\beta}}|y|^{1-\alpha} \mathrm{d} y \geqslant c_{d, \alpha} \int_{0}^{1 / 4} \frac{\mathrm{d} t}{t} .
$$

Hence, $\frac{\partial^{2}}{\partial x_{d}^{2}} w_{-}(0)=\infty$.

\section{Examples}

In the last section we present some examples of operators $\mathscr{L}$ resp. corresponding Dirichlet problems that allow for an application of Theorem 1.2. In Example 6.1 we modify the considerations from Section 5 in order to match the assumptions of Theorem 1.2. In Example 6.2 we generalize to subordinated Brownian motion. Finally, in Example 6.4 we extend 
the above class and discuss the process which is assumed only to have the lower scaling property on the characteristic exponent.

Example 6.1 (fractional Laplace operator) Let $X_{t}$ be strictly stable process whose generator is the fractional Laplace operator $-(-\Delta)^{\alpha / 2}$. Let $D$ be a bounded open set.

1. Let $\alpha \in(0,1)$. The potential kernel is of the form $G(y)=c_{d, \alpha}|y|^{\alpha-d}$ and satisfies

$$
\int_{0}^{1 / 2}\left|G^{\prime}(t)\right| t^{d-1} \mathrm{~d} t=\infty
$$

Here $S(r)=\left|G^{\prime}(r)\right|$. According to Theorem 1.2, there is a $C^{2}(D)$ solution of Eq. 1.7 if the following holds:

$$
\int_{0}^{1 / 2}\left|G^{\prime}(t)\right| \omega_{\nabla f}(t, D) t^{d-1} d t=c_{d, \alpha} \int_{0}^{1 / 2} t^{\alpha-2} \omega_{\nabla f}(t, D) d t<\infty .
$$

Obviously, our function from the counterexample $f(y)=\left(\left(y_{d}\right)_{+}\right)^{2-\alpha}$ which is $C^{2-\alpha}(D)$ does not satisfy (6.2). On the other hand, it is well known (see e.g. [39, Theorem 1.1(b)]) that for any function which is $C^{2-\alpha+\epsilon}, \epsilon>0$ (e.g. $\left.\tilde{f}(y)=\left(\left(y_{d}\right)_{+}\right)^{2-\alpha+\epsilon}\right)$, the solution of Eq. 1.7 is $C^{2}(D)$. Clearly, this function satisfies (6.2) as well, so in some sense Theorem 1.2 extends already known results. The sufficient condition is also $\omega_{\nabla f}(t, D) \leqslant C t^{1-\alpha} \ln ^{-\beta}\left(1+t^{-1}\right), \beta>1$. Then

$$
\begin{aligned}
\int_{0}^{1 / 2}\left|G^{\prime}(t)\right| \omega_{\nabla f}(t, D) t^{d-1} \mathrm{~d} t & \leqslant c \int_{0}^{1 / 2} t^{\alpha-2} t^{1-\alpha} \ln ^{-\beta}\left(1+t^{-1}\right) \mathrm{d} t \\
& \leqslant c \int_{0}^{1 / 2} t^{-1} \ln ^{-\beta}\left(t^{-1}\right) \mathrm{d} t \\
& =c \int_{\ln 2}^{\infty} \frac{\mathrm{d} t}{t^{\beta}}<\infty .
\end{aligned}
$$

Calculations in the cases below are very similar and therefore will be omitted.

2. Let $\alpha=d=1$. The compensated potential kernel is of the form $G(y)=\frac{1}{\pi} \ln \frac{1}{|y|}$ and Eq. 6.1 holds for $S(r)=\left|G^{\prime}(r)\right|$. Note that in this case $\left|G^{\prime}(r)\right| \neq c \frac{G(r)}{r}$. By Theorem 1.2 the solution of Eq. 1.7 will be in $C^{2}(D)$ if

$$
\int_{0}^{1 / 2}\left|G^{\prime}(t)\right| \omega_{\nabla f}(t, D) t^{d-1} \mathrm{~d} t=c \int_{0}^{1 / 2} t^{-1} \omega_{\nabla f}(t, D) \mathrm{d} t<\infty .
$$

Hence, it suffices that $\omega_{\nabla f}(t, D) \leqslant C \ln ^{-\beta}\left(1+t^{-1}\right), \beta>1$.

3. Let $\alpha=1, d>1$. The potential kernel has a form $G(y)=c_{d, \alpha}|y|^{1-d}$ and Eq. 6.1 holds for $S(r)=\left|G^{\prime}(r)\right|$. Analogously to the case $\alpha=d=1$ it suffices that $\omega_{\nabla f}(t, D) \leqslant$ $C \ln ^{-\beta}\left(1+t^{-1}\right), \beta>1$.

4. $\alpha \in(1,2), d=1$. The compensated potential kernel is of the form $G(y)=c_{\alpha}|y|^{\alpha-1}$, $S(r)=\left|G^{\prime \prime}(r)\right|$, and we have $\int_{0}^{1}\left|G^{\prime}(t)\right| \mathrm{d} t<\infty$, thus, by Theorem 1.2 , there will be a $C^{2}(D)$ solution if

$$
\int_{0}^{1 / 2}\left|G^{\prime \prime}(t)\right| \omega_{f}(t, D) t^{d-1} \mathrm{~d} t=c_{\alpha} \int_{0}^{1 / 2} t^{\alpha-3} \omega_{f}(t, D) \mathrm{d} t<\infty .
$$

Clearly the function $f(y)=\left(y_{+}\right)^{2-\alpha}$ from Section 5 does not satisfy (6.3). In order to correct it we may either take a function from $C^{2-\alpha+\epsilon}(D), \epsilon>0$ (e.g. $\tilde{f}(y)=$ 
$\left(y_{+}\right)^{2-\alpha+\epsilon}$ ) or a function whose modulus of continuity is of the form $\omega_{\tilde{f}}(t, D)=$ $t^{2-\alpha} \ln ^{-\beta}\left(1+t^{-1}\right), \beta>1$.

5. $\alpha \in(1,2), d \geqslant 2$. The potential kernel has the form $G(y)=c_{d, \alpha}|y|^{\alpha-d}$ and $S(r)=$ $\left|G^{\prime \prime}(r)\right|$. We have

$$
\int_{0}^{1 / 2}\left|G^{\prime}(t)\right| t^{d-1} \mathrm{~d} t<\infty .
$$

By Theorem 1.2 we may either take a function $\tilde{f}$ from $C^{2-\alpha+\epsilon}(D)$ or such that its modulus of continuity has the form $\omega_{\tilde{f}}(t, D)=t^{2-\alpha} \ln ^{-\beta}\left(1+t^{-1}\right), \beta>1$.

Example 6.2 (Subordinate Brownian motion) Let $\left(B_{t}, t \geqslant 0\right)$ be a Brownian motion in $\mathbb{R}^{d}$ and $\left(S_{t}, t \geqslant 0\right)$ - a subordinator independent from $B_{t}$, i.e. a Lévy process in $\mathbb{R}$ which starts from 0 and has non-decreasing trajectories. Process $\left(X_{t}, t \geqslant 0\right)$ defined by $X_{t}=B_{S_{t}}$ is called a subordinated Brownian motion. Denote by $\phi$ the Laplace exponent of $S_{t}$ :

$$
\mathbb{E} \exp \left\{-\lambda S_{t}\right\}=\exp \{-t \phi(\lambda)\} .
$$

It is well known (see [42] for a comprehensive study) that $\phi$ is of the form

$$
\phi(\lambda)=\gamma t+\int_{0}^{\infty}\left(1-e^{-\lambda t}\right) \mu(\mathrm{d} t)
$$

where $\mu$ is the Lévy measure of $S_{t}$ satisfying $\int_{0}^{\infty}(1 \wedge t) \mu(\mathrm{d} t)<\infty$. The corresponding operator is of the form $\mathscr{L}=-\phi(-\Delta)$ and we have $\psi(\xi)=\phi\left(|\xi|^{2}\right)$. An example of subordinated Brownian motion is the process from Example 6.1 with $\phi(\lambda)=\lambda^{\alpha / 2}, \alpha \in$ $(0,2)$. Another example is geometric stable process with $\phi(\lambda)=\ln \left(1+\lambda^{\alpha / 2}\right), \alpha \in(0,2)$. Denote by $G_{d}(r)$ the potential of $d$-dimensional subordinated Brownian motion $X_{t}$. From [22, Theorem 5.17] we have

$$
G_{d}(r) \asymp r^{-d-2} \frac{\phi^{\prime}\left(r^{-2}\right)}{\phi^{2}\left(r^{-2}\right)}, \quad r \rightarrow 0^{+},
$$

if $d \geqslant 3$ and there exist $\beta \in[0, d / 2+1)$ and $\alpha>0$ such that $\phi^{-2} \phi^{\prime}$ satisfies weak lower and upper scaling condition at infinity with exponents $-\beta$ and $-\alpha$, respectively (for details, see [22]). The same result under slightly stronger assumptions is derived in [30, Proposition 4.5].

For $d$-dimensional subordinated Brownian motion $X_{t}, d \geqslant 3$, we have

$$
G_{d}(r)=\int_{0}^{\infty}(4 \pi t)^{-d / 2} \exp \left(-\frac{r^{2}}{4 t}\right) u(\mathrm{~d} t),
$$

where $u(\mathrm{~d} t)$ is the potential measure of the subordinator. It follows that

$$
\begin{aligned}
G_{d}^{\prime}(r) & =-\int_{0}^{\infty}(4 \pi t)^{-d / 2} \exp \left(-\frac{r^{2}}{4 t}\right) \frac{2 r}{4 t} u(\mathrm{~d} t) \\
& =-2 r \pi \int_{0}^{\infty}(4 \pi t)^{-(d+2) / 2} \exp \left(-\frac{r^{2}}{4 t}\right) u(\mathrm{~d} t) \\
& =-2 r \pi G_{d+2}(r) .
\end{aligned}
$$

That and Eq. 6.4 imply

$$
\left|G_{d}^{\prime}(r)\right| \leqslant c r \cdot r^{-(d+2)-2} \frac{\phi^{\prime}\left(r^{-2}\right)}{\phi^{2}\left(r^{-2}\right)}=c \frac{1}{r} r^{-d-2} \frac{\phi^{\prime}\left(r^{-2}\right)}{\phi^{2}\left(r^{-2}\right)} \leqslant c \frac{G_{d}(r)}{r}, \quad r \rightarrow 0^{+} .
$$


By induction, for every $k \in \mathbb{N}$ there is $c_{k}>0$ such that

$$
\left|G_{d}^{(k)}(r)\right| \leqslant c_{k} \frac{G_{d}(r)}{r^{k}}, \quad r \rightarrow 0^{+} .
$$

Thus, the sufficient conditions involving $G$ and its derivatives hold true for $S(r)=G_{d}(r) / r$ or $S(r)=G_{d}(r) / r^{2}$, depending on finiteness of the expression $\int_{0}^{1 / 2} G_{d}^{\prime}(t) t^{d-1} \mathrm{~d} t$. Note that the density of Lévy measure of $X_{t}$,

$$
v(r)=\int_{0}^{\infty}(4 \pi t)^{-d / 2} \exp \left(-\frac{r^{2}}{4 t}\right) \mu(\mathrm{d} t),
$$

belongs to $C^{\infty}$. By [8, Lemma 7.4] the assumptions of Theorem 1.2 are satisfied with $v^{*} \equiv v$ if $\phi$ is a complete Bernstein function.

Take geometric stable process with $\phi(\lambda)=\ln \left(1+\lambda^{\alpha / 2}\right)$. Then, by Eqs. 6.4 and 6.5,

$$
\begin{aligned}
\int_{0}^{1 / 2}\left|G_{d}^{\prime}(t)\right| t^{d-1} \mathrm{~d} t & \geqslant c \int_{0}^{1 / 2} t^{-d-3} t^{d-1} \frac{\phi^{\prime}\left(t^{-2}\right)}{\phi^{2}\left(t^{-2}\right)} \mathrm{d} t=c \int_{0}^{1 / 2} \frac{1}{t^{4}} \frac{\frac{1}{1+t^{-\alpha}} \frac{1}{\ln ^{2}\left(1+t^{-\alpha}\right)}}{\mathrm{d} t} \\
& \geqslant c \int_{0}^{1 / 2} \frac{1}{t^{2}} \frac{1}{\ln ^{2}\left(1+t^{-\alpha}\right)} \mathrm{d} t \geqslant c \int_{0}^{1 / 2} \frac{\mathrm{d} t}{t^{2}}=\infty,
\end{aligned}
$$

hence, for the solution of Eq. 1.7 to be in $C^{2}(D)$, it suffices that the modulus of continuity of gradient of function $f$ is of the form $\omega_{\nabla f}(t, D)=t \ln ^{1-\epsilon}\left(1+t^{-1}\right), \epsilon \in(0,1)$.

Before moving to the last example, let us define concentration functions $K$ and $h$ by setting

$$
\begin{aligned}
& K(r)=\frac{1}{r^{2}} \int_{|x| \leqslant r}|x|^{2} v(\mathrm{~d} x), \quad r>0, \\
& h(r)=\int_{\mathbb{R}^{d}}\left(1 \wedge \frac{|x|^{2}}{r^{2}}\right) v(\mathrm{~d} x), \quad r>0 .
\end{aligned}
$$

We note that in our setting, $h$ is strictly decreasing.

Proposition 6.3 Let $d \geqslant 3$. Suppose there exist $c>0$ and $\alpha \geqslant 3 / 2$ such that

$$
h(r) \leqslant c \lambda^{\alpha} h(\lambda r), \quad \lambda \leqslant 1, r>0 .
$$

Then there exists $c>0$ such that $\left|U^{\prime}(r)\right| \leqslant c U(r) / r,\left|U^{\prime \prime}(r)\right| \leqslant c U(r) / r^{2},\left|U^{\prime \prime \prime}(r)\right| \leqslant$ $c U(r) / r^{3}$ for $r>0$.

Proof Observe that for $d \geqslant 3$ the potential $U$ always exists (see e.g. [41, Theorem 37.8]). By [19, Corollary 1 and Theorem 3] there exists $c>0$ such that

$$
U(r) \geqslant \frac{c}{|r|^{d} h(r)}, \quad r>0 .
$$

Our aim is to prove $(\mathrm{G})$. By definition and isotropy of $p_{t}$,

$$
U(r)=\int_{0}^{\infty} p_{t}(\tilde{r}) \mathrm{d} t
$$

where $\tilde{r}=(0, \ldots, 0, r) \in \mathbb{R}^{d}$. Since $p_{t}$ is radially decreasing, by the Tonelli theorem,

$$
U(r)-U(1)=\int_{0}^{\infty} \int_{1}^{r} \partial_{x_{d}} p_{t}(\tilde{y}) \mathrm{d} y \mathrm{~d} t=\int_{1}^{r} \int_{0}^{\infty} \partial_{x_{d}} p_{t}(\tilde{y}) \mathrm{d} t \mathrm{~d} y,
$$


where $\tilde{y}=(0, \ldots, 0, y) \in \mathbb{R}^{d}$. Hence,

$$
U^{\prime}(r)=\int_{0}^{\infty} \partial_{x_{d}} p_{t}(\tilde{r}) \mathrm{d} t, \quad r>0 .
$$

By [23, Theorem 5.6 and Corollary 6.8],

$$
\left|\partial_{x}^{\beta} p_{t}(x)\right| \leqslant c\left(h^{-1}(1 / t)\right)^{-|\beta|} \varphi_{t}(x), \quad t>0, x \in \mathbb{R}^{d},
$$

where

$$
\varphi_{t}(x)= \begin{cases}\left(h^{-1}(1 / t)\right)^{-d}, & |x| \leqslant h^{-1}(1 / t), \\ t K(|x|)|x|^{-d}, & |x|>h^{-1}(1 / t) .\end{cases}
$$

Let us estimate $\left|U^{\prime}(r)\right|$. We have

$$
\left|U^{\prime}(r)\right| \leqslant c \frac{K(r)}{r^{d}} \int_{0}^{1 / h(r)} \frac{t}{h^{-1}(1 / t)} \mathrm{d} t+c \int_{1 / h(r)}^{\infty} \frac{\mathrm{d} t}{\left(h^{-1}(1 / t)\right)^{d+1}} .
$$

The scaling property of $h$ for $r>h^{-1}(1 / t)$ yields

$$
h(r) \leqslant c\left(\frac{h^{-1}(1 / t)}{r}\right)^{\alpha} h\left(h^{-1}(1 / t)\right) .
$$

It follows that

$$
\begin{aligned}
\frac{K(r)}{r^{d}} \int_{0}^{1 / h(r)} \frac{t}{h^{-1}(1 / t)} \mathrm{d} t & \leqslant c \frac{K(r)}{r^{d+1}} \int_{0}^{1 / h(r)} t\left(\frac{1}{t h(r)}\right)^{1 / \alpha} \mathrm{d} t \\
& \leqslant c \frac{K(r)}{r^{d+1}}(h(r))^{-1 / \alpha} \int_{0}^{1 / h(r)} t^{1-1 / \alpha} \mathrm{d} t .
\end{aligned}
$$

For $\alpha>1 / 2$ the integral is finite and we get

$$
\frac{K(r)}{r^{d}} \int_{0}^{1 / h(r)} \frac{t}{h^{-1}(1 / t)} \mathrm{d} t \leqslant c \frac{K(r)}{r^{d+1} h(r)^{2}} .
$$

The comparability of $K$ and $h$ ([24, Lemma 2.3]) implies

$$
\frac{K(r)}{r^{d}} \int_{0}^{1 / h(r)} \frac{t}{h^{-1}(1 / t)} \mathrm{d} t \leqslant c \frac{1}{r^{d+1} h(r)} \leqslant c \frac{U(r)}{r} .
$$

Furthermore, we always have $h(r) \geqslant \lambda^{2} h(\lambda r)$ for $\lambda \leqslant 1$ and $r>0$. Thus,

$$
\begin{aligned}
\int_{1 / h(r)}^{\infty} \frac{\mathrm{d} t}{\left(h^{-1}(1 / t)\right)^{d+1}} & =\frac{1}{r^{d+1}} \int_{1 / h(r)}^{\infty} \frac{r^{d+1}}{\left(h^{-1}(1 / t)\right)^{d+1}} \mathrm{~d} t \\
& \leqslant \frac{1}{r^{d+1}} \int_{1 / h(r)}^{\infty}\left(\frac{1}{t h(r)}\right)^{(d+1) / 2} \mathrm{~d} t .
\end{aligned}
$$

Since $d>1$, the integral is finite and we get

$$
\int_{1 / h(r)}^{\infty} \frac{\mathrm{d} t}{\left(h^{-1}(1 / t)\right)^{d+1}} \leqslant c \frac{1}{r^{d+1} h(r)} \leqslant c \frac{U(r)}{r} .
$$

Hence, for $\alpha>1 / 2$ we obtain $\left|U^{\prime}(r)\right| \leqslant c U(r) / r, r>0$. By similar argument one may conclude that $\left|U^{\prime \prime}(r)\right| \leqslant c U(r) / r^{2}$ if $\alpha>1$ and $\left|U^{\prime \prime \prime}(r)\right| \leqslant c U(r) / r^{3}$ for $\alpha>3 / 2$.

Example 6.4 Let $d \geqslant 3, \alpha>3 / 2$, and $X_{t}$ be a truncated $\alpha$-stable Lévy process in $\mathbb{R}^{d}$, i.e. with Lévy measure $v(x)=|x|^{-d-\alpha} \varphi(x)$, where $\varphi$ is a cut-off function, i.e. $\varphi \in C^{\infty}\left(\mathbb{R}^{d}\right)$ 
and $\mathbf{1}_{B_{1 / 2}} \leqslant \varphi \leqslant \mathbf{1}_{B_{1}}$. One can easily check that $h(r) \asymp r^{-\alpha} \wedge r^{-2}$. Proposition 6.3 yields that the assumptions of Theorem 1.2 imposed on function $G$ are satisfied. Observe that (A) and Eq. 1.2 is satisfied for $v^{*} \equiv v$ and any $r_{0} \geqslant 1$. In that case the appropriate $\mathcal{L}^{1}$ space is simply $L_{\text {loc }}^{1}$.

Acknowledgments We would like to express our deep gratitude to the anonymous reviewers for numerous helpful and valuable comments and suggestions which significantly contributed to the quality of this article.

Open Access This article is licensed under a Creative Commons Attribution 4.0 International License, which permits use, sharing, adaptation, distribution and reproduction in any medium or format, as long as you give appropriate credit to the original author(s) and the source, provide a link to the Creative Commons licence, and indicate if changes weremade. The images or other third party material in this article are included in the article's Creative Commons licence, unless indicated otherwise in a credit line to the material. If material is not included in the article's Creative Commons licence and your intended use is not permitted by statutory regulation or exceeds the permitted use, you will need to obtain permission directly from the copyright holder. To view a copy of this licence, visit http://creativecommons.org/licenses/by/4.0/.

\section{Appendix A: Potential Theory for Recurrent Unimodal Lévy Processes}

In this appendix we establish a formula for the Green function for a bounded open set $D$ in case of recurrent isotropic unimodal Lévy process $X_{t}$. Contrary to the transient case, here the potential kernel $U(x)=\int_{0}^{\infty} p_{t}(x) \mathrm{d} t$ is infinite (see [41, Chapter 7] for a detailed discussion on the subject), so the classical Hunt formula has no application. Instead, one can define the $\lambda$-potential kernel $U^{\lambda}$ by setting

$$
U^{\lambda}(x)=\int_{0}^{\infty} e^{-\lambda t} p_{t}(x) \mathrm{d} t, \quad \lambda \geqslant 0, x \in \mathbb{R}^{d} .
$$

Similarly, we define the $\lambda$-Green function for an open set $D$

$$
G_{D}^{\lambda}(x, y)=\int_{0}^{\infty} e^{-\lambda t} p_{t}^{D}(x, y) \mathrm{d} t, \quad \lambda \geqslant 0, x, y \in \mathbb{R}^{d} .
$$

Note that both $U^{\lambda}$ and $G_{D}^{\lambda}$ exist. An analogue of the Hunt formula for $G_{D}^{\lambda}$ holds, namely, for $x, y \in \mathbb{R}^{d}$,

$$
G_{D}^{\lambda}(x, y)=U^{\lambda}(y-x)-\mathbb{E}^{x}\left[e^{-\lambda \tau_{D}} U^{\lambda}\left(y-X_{\tau_{D}}\right)\right] .
$$

Lemma A.1 Let $d \geqslant 1$. For any fixed $x_{0} \in \mathbb{R}^{d} \backslash\{0\}$ we have $\lambda U^{\lambda}\left(x_{0}\right) \rightarrow 0$ as $\lambda \rightarrow 0^{+}$.

Proof In the following part we introduce a mild ambiguity by denoting by 1 , depending on the context, either a real number or the vector $(0, \ldots, 0,1) \in \mathbb{R}^{d}$. Set $x_{0}=1$. Let $f_{\lambda}(r)=$ $\int_{|x|<\sqrt{r}} \mathrm{~d} x \int_{0}^{\infty} e^{-\lambda u} p_{u}(x) \mathrm{d} u$. We have

$$
\begin{aligned}
L f_{\lambda}(s) & =\int_{0}^{\infty} e^{-s t} f_{\lambda}(t) \mathrm{d} t=\int_{0}^{\infty} e^{-s t} \int_{|x|<\sqrt{t}} \mathrm{~d} x \int_{0}^{\infty} e^{-\lambda u} p_{u}(x) \mathrm{d} u \mathrm{~d} t \\
& =\int_{\mathbb{R}^{d}} \mathrm{~d} x \int_{t>|x|^{2}} e^{-s t} \mathrm{~d} t \int_{0}^{\infty} e^{-\lambda u} p_{u}(x) \mathrm{d} u=\frac{1}{s} \int_{0}^{\infty} e^{-\lambda u} \int_{\mathbb{R}^{d}} e^{-s|x|^{2}} p_{u}(x) \mathrm{d} x \mathrm{~d} u
\end{aligned}
$$

By the proof of [19, Lemma 6],

$$
\int_{\mathbb{R}^{d}} e^{-s|x|^{2}} p_{u}(x) \mathrm{d} x=c_{d} \int_{\mathbb{R}^{d}} e^{-u \psi(\sqrt{s} x)} e^{-|x|^{2} / 4} \mathrm{~d} x .
$$


Hence, we have for $\lambda>0$,

$s L f_{\lambda}(s)=c_{d} \int_{0}^{\infty} e^{-\lambda u} \mathrm{~d} u \int_{\mathbb{R}^{d}} e^{-u \psi(\sqrt{s} \xi)} e^{-|\xi|^{2} / 4} \mathrm{~d} \xi=c_{d} \int_{\mathbb{R}^{d}} \frac{1}{\lambda+\psi(\sqrt{s} \xi)} e^{-|\xi|^{2} / 4} \mathrm{~d} \xi$.

By monotonicity of $f_{\lambda}$,

$$
\begin{aligned}
f_{\lambda}(r) & =\frac{e}{r} \int_{r}^{\infty} e^{-u / r} f_{\lambda}(r) \mathrm{d} u \leqslant \frac{e}{r} \int_{0}^{\infty} e^{-u / r} f_{\lambda}(u) \mathrm{d} u=\frac{e}{r} L f_{\lambda}(1 / r) \\
& =c^{\prime} \int_{\mathbb{R}^{d}} \frac{1}{\lambda+\psi(\sqrt{1 / r} \xi)} e^{-|\xi|^{2} / 4} \mathrm{~d} \xi .
\end{aligned}
$$

Since by [19, Lemma 1 and Proposition 1],

$$
\sup _{|x| \leqslant 1} \psi(x) \leqslant \frac{4}{|\xi|^{2}} \sup _{|x| \leqslant|\xi|} \psi(x) \leqslant c \frac{\psi(\xi)}{|\xi|^{2}}, \quad|\xi| \leqslant 1
$$

we obtain

$$
\begin{aligned}
\lambda U^{\lambda}(1) \leqslant \lambda \frac{f_{\lambda}(1)}{\left|B_{1}\right|} & \leqslant c_{d} \int_{\mathbb{R}^{d}} \frac{\lambda}{\lambda+\psi(\xi)} e^{-|\xi|^{2} / 4} \mathrm{~d} \xi \\
& \leqslant \tilde{c}\left(\frac{\lambda}{\psi(1)} \int_{B_{1}^{c}} e^{-|\xi|^{2} / 4} d \xi+\int_{B_{1}} \frac{\lambda}{\lambda+|\xi|^{2}} \mathrm{~d} \xi\right) .
\end{aligned}
$$

Hence, $\lambda U^{\lambda}(1) \rightarrow 0$ as $\lambda \rightarrow 0^{+}$. The extension to arbitrary $x_{0}$ is immediate.

Lemma A.2 Let $x_{0} \in \mathbb{R}^{d} \backslash\{0\}$ be an arbitrary fixed point. For all $x \in \mathbb{R}^{d} \backslash\{0\}$ we have $\int_{0}^{\infty}\left|p_{t}(x)-p_{t}\left(x_{0}\right)\right| \mathrm{d} t<\infty$.

Proof Let $f \in C_{c}^{\infty}\left(\mathbb{R}^{d}\right)$ be symmetric and such that $\mathbf{1}_{B_{\epsilon}} \leqslant f \leqslant \mathbf{1}_{B_{4} \epsilon}$, where $0<4 \epsilon<1$. Denote, for $\lambda>0$,

$$
\begin{aligned}
& W_{x_{0}}^{\lambda}(x)=\int_{0}^{\infty} e^{-\lambda t}\left(p_{t}(x)-p_{t}\left(x_{0}\right)\right) \mathrm{d} t, \quad x \neq 0, \\
& W_{x_{0}}(x)=\int_{0}^{\infty}\left(p_{t}(x)-p_{t}\left(x_{0}\right)\right) \mathrm{d} t, \quad x \neq 0 .
\end{aligned}
$$

Let $x_{0}=1$. Observe that

$$
W_{1}^{\lambda} * f(0)=\int_{0}^{\infty} e^{-\lambda t}\left(p_{t} * f(0)-p_{t}(1)\|f\|_{1}\right) \mathrm{d} t .
$$

Note that the integrand has a positive sign. Indeed,

$$
p_{t} * f(0)-p_{t}(1)\|f\|_{1}=\int_{B_{4 \epsilon}}\left(p_{t}(y) f(y)-p_{t}(1) f(y)\right) \mathrm{d} y>0,
$$

since $4 \epsilon<1$. Furthermore,

$$
p_{t}(1)\|f\|_{1}=\int_{B_{4 \epsilon}} p_{t}(1) f(y) \mathrm{d} y \geqslant \int_{B_{4 \epsilon}} p_{t}(1+4 \epsilon-y) f(y) \mathrm{d} y=p_{t} * f(1+4 \epsilon) .
$$


Hence, by the Fourier inversion theorem,

$$
\begin{aligned}
\int_{0}^{\infty} e^{-\lambda t}\left(p_{t} * f(0)-p_{t}(1)\|f\|_{1}\right) \mathrm{d} t & \leqslant \int_{0}^{\infty} e^{-\lambda t} \int_{\mathbb{R}^{d}}(1-\cos ((1+4 \epsilon) \xi)) \widehat{p_{t}}(\xi)|\widehat{f}(\xi)| \mathrm{d} \xi \mathrm{d} t \\
& \leqslant \int_{\mathbb{R}^{d}}(1-\cos ((1+4 \epsilon) \xi)) \frac{|\widehat{f}(\xi)|}{\psi(\xi)} \mathrm{d} \xi
\end{aligned}
$$

By the monotone convergence theorem, Eq. A.1 and the fact that $|\widehat{f}(\xi)|$ decays faster than any polynomial,

$$
W_{1} * f(0)=\lim _{\lambda \rightarrow 0} W_{1}^{\lambda} * f(0) \leqslant \int_{\mathbb{R}^{d}}(1-\cos ((1+4 \epsilon) \xi)) \frac{|\widehat{f}(\xi)|}{\psi(\xi)} \mathrm{d} \xi<\infty .
$$

Hence,

$$
\int_{B_{\epsilon}} W_{1}(x) \mathrm{d} x \leqslant W_{1} * f(0)<\infty .
$$

Since $W_{1}$ is radially decreasing and positive for $|x|<1$, Eq. A.2 implies that it may be infinite only for $x=0$. It follows that $W_{1}$ is well defined for $0<|x| \leqslant 1$. Similarly $0 \leqslant W_{x_{0}}<\infty$ for $0<|x| \leqslant\left|x_{0}\right|$.

It remains to notice that for $|x|>\left|x_{0}\right|$ we have $0 \leqslant\left|W_{x_{0}}(x)\right|=-W_{x_{0}}(x)=W_{x}\left(x_{0}\right)<$ $\infty$ by the first part of the proof.

Lemma A.2 allows us to introduce, following [5], [10], [28], a compensated potential kernel by setting for $x \in \mathbb{R}^{d} \backslash\{0\}$

$$
W_{x_{0}}(x):=\int_{0}^{\infty}\left(p_{t}(x)-p_{t}\left(x_{0}\right)\right) \mathrm{d} t
$$

where $x_{0} \in \mathbb{R}^{d} \backslash\{0\}$ is an arbitrary but fixed point. From the proof of Lemma A.2 we immediately obtain the following corollary.

Corollary A.3 For any $x_{0} \in \mathbb{R}^{d} \backslash\{0\}, W_{x_{0}}$ is locally integrable in $\mathbb{R}^{d}$.

Theorem A.4 Let $x_{0} \in \mathbb{R}^{d} \backslash\{0\}, d \leqslant 2$ and $D$ be bounded. Then for $x, y \in D$,

$$
G_{D}(x, y)=W_{x_{0}}(y-x)-\mathbb{E}^{x} W_{x_{0}}\left(y-X_{\tau_{D}}\right) .
$$

Proof Let $x, y \in D$. Fix $x_{0} \in \mathbb{R}^{d}\{0\}$ and observe that for any $\lambda>0$,

$$
\begin{aligned}
G_{D}^{\lambda}(x, y)= & U^{\lambda}(y-x)-\mathbb{E}^{x}\left[e^{-\lambda \tau_{D}} U^{\lambda}\left(y-X_{\tau_{D}}\right)\right] \\
= & U^{\lambda}(x-y)-U^{\lambda}\left(x_{0}\right)-\mathbb{E}^{x}\left[e^{-\lambda \tau_{D}}\left(U^{\lambda}\left(y-X_{\tau_{D}}\right)-U^{\lambda}\left(x_{0}\right)\right)\right] \\
& +U^{\lambda}\left(x_{0}\right) \mathbb{E}^{x}\left[1-e^{-\lambda \tau_{D}}\right] .
\end{aligned}
$$

We want to pass with $\lambda$ to $0^{+}$. The limit of left-hand side is well defined and is equal to $G_{D}(x, y)$, which, in view of [20, Theorem 1.3], is finite. From Lemma A.1 we get

$$
U^{\lambda}\left(x_{0}\right) \mathbb{E}^{x}\left[1-e^{-\lambda \tau_{D}}\right] \leqslant \lambda U^{\lambda}\left(x_{0}\right) \sup _{x \in \mathbb{R}^{d}} \mathbb{E}^{x} \tau_{D} \stackrel{\lambda \rightarrow 0^{+}}{\longrightarrow} 0 .
$$

Moreover, from Lemma A.2 we obtain that

$$
\lim _{\lambda \rightarrow 0^{+}}\left(U^{\lambda}(y-x)-U^{\lambda}\left(x_{0}\right)\right)=W_{x_{0}}(y-x) .
$$


It remains to show the convergence of the middle term of Eq. A.5. Since $U^{\lambda}$ is radially decreasing, $U^{\lambda}\left(y-X_{\tau_{D}}\right)-U^{\lambda}\left(x_{0}\right)$ is positive and increasing as $\lambda \rightarrow 0^{+}$on the set $\{y \in$ $\left.\mathbb{R}^{d}:\left|y-X_{\tau_{D}}\right| \leqslant\left|x_{0}\right|\right\}$, and non-positive on its complement. By Lemma A.2, and the Monotone Convergence Theorem,

$$
\begin{aligned}
& \lim _{\lambda \rightarrow 0^{+}} \mathbb{E}^{x}\left[e^{-\lambda \tau_{D}}\left(U^{\lambda}\left(y-X_{\tau_{D}}\right)-U^{\lambda}\left(x_{0}\right)\right) ;\left|y-X_{\tau_{D}}\right|<\left|x_{0}\right|\right] \\
= & \mathbb{E}^{x}\left[W_{x_{0}}\left(y-X_{\tau_{D}}\right) ;\left|y-X_{\tau_{D}}\right|<\left|x_{0}\right|\right] \leqslant W_{x_{0}}\left(\delta_{D}(y)\right)<\infty .
\end{aligned}
$$

Observe that the left-hand side of Eq. A.5 converges to $G_{D}$ so it is finite. The remaining integral on the right-hand side converges as well by the monotone convergence theorem, but since all the other terms are finite, it follows that the integral is also finite and we obtain

$$
\lim _{\lambda \rightarrow 0^{+}} \mathbb{E}^{x}\left[e^{-\lambda \tau_{D}}\left(U^{\lambda}\left(y-X_{\tau_{D}}\right)-U^{\lambda}\left(x_{0}\right)\right)\right]=\mathbb{E}^{x} W_{x_{0}}\left(y-X_{\tau_{D}}\right),
$$

which ends the proof.

\section{References}

1. Abatangelo, N., Jarohs, S., Saldaña, A.: Green function and Martin kernel for higher-order fractional Laplacians in balls. Nonlinear Analysis 175, 173-190 (2018)

2. Bae, J., Kassmann, M.: Schauder estimates in generalized Hölder spaces. Preprint, 2015, arXiv:1505.05498 (2015)

3. Bass, R.F.: Regularity results for stable-like operators. J. Funct. Anal. 257(8), 2693-2722 (2009)

4. Bertoin, J.: Lévy Processes. Cambridge Tracts in Mathematics, vol. 121. Cambridge University Press, Cambridge (1996)

5. Blumenthal, R.M., Getoor, R.K., Ray, D.B.: On the distribution of first hits for the symmetric stable processes. Trans. Amer. Math. Soc. 99, 540-554 (1961)

6. Bogdan, K.: Representation of $\alpha$-harmonic functions in Lipschitz domains. Hiroshima Math. J. 29(2), 227-243 (1999)

7. Bogdan, K., Byczkowski, T.: Potential theory for the $\alpha$-stable Schrödinger operator on bounded Lipschitz domains. Studia Math. 133(1), 53-92 (1999)

8. Bogdan, K., Grzywny, T., Pietruska-Pałuba, K., Rutkowski, A.: Extension and trace for nonlocal operators. To appear in J. Math. Pure Appl. https://doi.org/10.1016/j.matpur.2019.09.005 (2019)

9. Bogdan, K., Grzywny, T., Ryznar, M.: Barriers, exit time and survival probability for unimodal Lévy processes. Probab. Theory Related Fields 162(1-2), 155-198 (2015)

10. Bogdan, K., Żak, T.: On Kelvin transformation. J. Theoret. Probab. 19(1), 89-120 (2006)

11. Bucur, C.: Some observations on the Green function for the ball in the fractional Laplace framework. Commun. Pure Appl. Anal. 15(2), 657-699 (2016)

12. Burch, C.C.: The Dini condition and regularity of weak solutions of elliptic equations. J. Differential Equations 30(3), 308-323 (1978)

13. Chung, K.L., Zhao, Z.X.: From Brownian Motion to Schrödinger's Equation, vol 312 of Grundlehren der Mathematischen Wissenschaften [Fundamental Principles of Mathematical Sciences]. Springer, Berlin (1995)

14. Duzaar, F., Gastel, A., Mingione, G.: Elliptic systems, singular sets and Dini continuity. Comm. Partial Differential Equations 29(7-8), 1215-1240 (2004)

15. Dyda, B., Kassmann, M.: Function spaces and extension results for nonlocal dirichlet problems. J. Funct. Anal. 277(11), 108-134 (2019)

16. Dynkin, E.B.: Markov processes. Vols. I, II, vol 122 of Translated with the authorization and assistance of the author by J. Fabius, V. Greenberg, A. Maitra, G. Majone. Die Grundlehren der Mathematischen Wissenschaften, Bände 121. Academic Press Inc., Publishers, New York; Springer, Berlin (1965)

17. Felsinger, M., Kassmann, M., Voigt, P.: The Dirichlet problem for nonlocal operators. Math. Z. 279(3-4), 779-809 (2015)

18. Grubb, G.: Local and nonlocal boundary conditions for $\mu$-transmission and fractional elliptic pseudodifferential operators. Anal. PDE 7(7), 1649-1682 (2014)

19. Grzywny, T.: On Harnack inequality and Hölder regularity for isotropic unimodal Lévy processes. Potential Anal. 41(1), 1-29 (2014) 
20. Grzywny, T., Kwaśnicki, M.: Potential kernels, probabilities of hitting a ball, harmonic functions and the boundary Harnack inequality for unimodal Lévy processes. Stochastic Process. Appl. 128(1), 1-38 (2018)

21. Grzywny, T., Ryznar, M.: Hitting times of points and intervals for symmetric Lévy processes. Potential Anal. 46(4), 739-777 (2017)

22. Grzywny, T., Ryznar, M., Trojan, B.: Asymptotic behaviour and estimates of slowly varying convolution semigroups. International Mathematics Research Notices 2019(23), 7193-7258 (2019)

23. Grzywny, T., Szczypkowski, K.: Estimates of heat kernel for non-symmetric Lévy processes. Preprint, 2017, arXiv:1710.07793 (2017)

24. Grzywny, T., Szczypkowski, K.: Lévy processes: concentration function and heat kernel bounds. Preprint, 2019, arXiv:1907.00778 (2019)

25. Grzywny, T., Szczypkowski, K.: Kato classes for Lévy processes. Potential Anal. 47(3), 245-276 (2017)

26. Hartman, P., Wintner, A.: On uniform Dini conditions in the theory of linear partial differential equations of elliptic type. Amer. J. Math. 77, 329-354 (1955)

27. Ikeda, N., Watanabe, S.: On some relations between the harmonic measure and the Lévy measure for a certain class of Markov processes. J. Math. Kyoto Univ. 2, 79-95 (1962)

28. Kac, M.: Some remarks on stable processes. Publ. Inst. Statist. Univ. Paris 6, 303-306 (1957)

29. Kim, M., Kim, P., Lee, J., Lee, K.-A.: Boundary regularity for nonlocal operators with kernels of variable orders. J. Funct. Anal. 277(1), 279-332 (2019)

30. Kim, P., Mimica, A.: Harnack inequalities for subordinate Brownian motions. Electron. J. Probab. 17(37), 1-23 (2012)

31. Kovats, J.: Fully nonlinear elliptic equations and the Dini condition. Comm. Partial Differential Equations 22(11-12), 1911-1927 (1997)

32. Kulczycki, T., Ryznar, M.: Gradient estimates of harmonic functions and transition densities for Lévy processes. Trans. Amer. Math. Soc. 368(1), 281-318 (2016)

33. Matiı̌čuk, M.I., Ė̌̆del'man, S.D.: Boundary value problems for second order parabolic and elliptic equations in Dini spaces. Dokl. Akad. Nauk SSSR 198, 533-536 (1971)

34. McShane, E.J.: Extension of range of functions. Bull. Amer. Math. Soc. 40(12), 837-842 (1934)

35. Pruitt, W.E.: The growth of random walks and Lévy processes. Ann. Probab. 9(6), 948-956 (1981)

36. Ros-Oton, X.: Nonlocal elliptic equations in bounded domains: a survey. Publ. Mat. 60(1), 3-26 (2016)

37. Ros-Oton, X., Serra, J.: The Dirichlet problem for the fractional Laplacian: regularity up to the boundary. J. Math. Pures Appl. 101(3), 275-302 (2014)

38. Ros-Oton, X., Serra, J.: Boundary regularity for fully nonlinear integro-differential equations. Duke Math. J. 165(11), 2079-2154 (2016)

39. Ros-Oton, X., Serra, J.: Regularity theory for general stable operators. J. Differential Equations 260(12), 8675-8715 (2016)

40. Rutkowski, A.: The Dirichlet problem for nonlocal Lévy-type operators. Publ. Mat. 62(1), 213-251 (2018)

41. Sato, K.-I.: Lévy Processes and Infinitely Divisible Distributions, vol 68 of Cambridge Studies in Advanced Mathematics. Cambridge University Press, Cambridge (1999). Translated from the 1990 Japanese original, Revised by the author

42. Schilling, R.L., Song, R., Vondraček, Z.: Bernstein functions: Theory and applications, vol 37 of de Gruyter Studies in Mathematics. 2nd edn. (2019)

43. Sztonyk, P.: On harmonic measure for Lévy processes. Probab. Math. Statist. 20(2), 383-390 (2000)

44. Watanabe, T.: The isoperimetric inequality for isotropic unimodal Lévy processes. Z. Wahrsch. Verw. Gebiete 63(4), 487-499 (1983)

45. Zhao, Z.: A probabilistic principle and generalized Schrödinger perturbation. J. Funct. Anal. 101(1), 162-176 (1991)

Publisher's Note Springer Nature remains neutral with regard to jurisdictional claims in published maps and institutional affiliations. 